\title{
Hypercapnia promotes microglial pyroptosis via inhibiting mitophagy: implication in neuroinflammation in hypoxemic adult rats
}

Hong-Guang Ding

Guangdong Provincial People's Hospital and Guangdong Academy of Medical Sciences

\section{Xu-Sheng Li}

Guangdong Provincial People's Hospital and Guangdong Academy of Medical Sciences

Xin-Qiang Liu

Guangdong Provincial People's Hospital and Guangdong Academy of Medical Sciences

Kang-Rong Wang

Southern Medical University

Ya Li

South China University of Technology

Miao-Yun Wen

Guangdong Provincial People's Hospital and Guangdong Academy of Medical Sciences

Hong-Ke Zeng ( $\nabla$ zenghongke@vip.163.com )

Guangdong Provincial People's Hospital and Guangdong Academy of Medical Sciences

https://orcid.org/0000-0003-3243-4199

\section{Research}

Keywords: ARDS, hypercapnia, hypoxemia, mitophagy, pyroptosis

Posted Date: December 31st, 2019

DOI: https://doi.org/10.21203/rs.2.19794/v1

License: (c) (1) This work is licensed under a Creative Commons Attribution 4.0 International License.

Read Full License 


\section{Abstract}

Background: Hypoxemia is a typical symptom of acute respiratory distress syndrome (ARDS).

Ventilations are always needed for correcting hypoxemia. To avoid pulmonary morbidity, low tidal volume ventilation is often applied. The ventilation strategy will certainly result in hypercapnia. Our previous study found that hypercapnia could induce IL-1 $\beta$ overproduction in hypoxia-activated microglia. However, the underlying mechanism has remained unclear. This study aimed to explore whether hypercapnia would promote microglial pyroptosis via inhibiting mitophagy in adult rats with hypoxemia.

Methods: Cerebral oxygen extraction ratio $\left(\mathrm{CERO}_{2}\right)$, partial pressure of brain tissue oxygen $\left(\mathrm{PbtO}_{2}\right)$, and reactive oxygen species (ROS) production in brain tissue in a rat model of hypercapnia/hypoxemia were evaluated. Along with this, the oxygen consumption rate (OCR) and ROS production of BV-2 microglial cells were evaluated after $15 \% \mathrm{CO}_{2} / 0.2 \% \mathrm{O}_{2}$ treatment. Mitophagy was observed using transmission electron microscopy (TEM). The protein expression level of LC3-II/I, p62, caspase-1, gasdermin D-N domains (GSDMD-N), IL-1 $\beta$ and IL-18 in microglial cells were detected before and after application of a ROS scavenger in vivo and in vitro.

Results: $\mathrm{PbtO}_{2}$ level was elevated by hypercapnia in the hypoxemic rats in the first $1.5 \mathrm{~h}$, but it was significantly decreased $2 \mathrm{~h}$ after ventilation. This was further evident by the increased levels of $\mathrm{CERO}_{2}$ at $3 \mathrm{~h}$ after ventilation. Besides, a high concentration of $\mathrm{CO}_{2}$ treatment could increase the levels of OCR in hypoxic BV-2 microglial cells in vitro. Expression levels of LC3-II were reduced, while those of p62 were increased by hypercapnia in the hypoxic hippocampus and BV-2 microglia. Autophagosomes could be seen in hypoxia treated microglial cells under TEM, while those were rarely observed in hypercapnia + hypoxia treated microglia. Hypercapnia markedly increased the production of ROS and the expression of caspase-1, GSDMD-N, IL-1 $\beta$, and IL-18 in hypoxia-activated microglia both in vivo and in vitro. Pharmacological scavenging ROS inhibited microglial pyroptosis and expression of IL-1 $\beta$ and IL-18.

Conclusion: Hypercapnia-induced mitophagy inhibition may promote pyroptosis and enhance IL-1 $\beta$ and IL-18 release in hypoxia-activated microglia.

\section{Background}

Acute respiratory distress syndrome (ARDS) is a common critical disease in intensive care units (ICUs) [1, 2]. Persistent hypoxemia is a typical symptom of ARDS. Ventilations are always needed to correct hypoxemia[3, 4] To avoid or reduce pulmonary morbidity, the low tidal volume ventilation is often applied $[5,6]$. The ventilation strategy will certainly result in hypercapnia, which is called "permissive hypercapnia" $[7,8]$. Our previous study found that hypercapnia could induce IL-1 $\beta$ overproduction in hypoxia-activated microglia[9]. However, the underlying mechanism has remained unclear.

The NLRP3 inflammasome is widely expressed in microglia, which plays an important role in initiating the central immune and inflammatory response[10-12]. To exert its functions, the NLRP3 inflammasome 
requires to be activated. The NLRP3 inflammasome can be triggered by an array of stimuli, including ATP, potassium efflux, heme, urate, and reactive oxygen species (ROS)[13-17]. Activation of the NLRP3 inflammasome can upregulate the expression of caspase- 1 and then leads to interleukin (IL)-18 and IL-1 $\beta$ maturation and pyroptosis activation. Pyroptosis is a proinflammatory cell death[18]. Recent studies have reported that gasdermin $\mathrm{D}$ (GSDMD) is the executioner of pyroptosis[19]. After being cleaved by caspase1 , gasdermin D-N domains (GSDMD-N) can form a pore in the plasma membrane. IL-1 $\beta$ and IL-18 are released through the pores, thereby perpetuating cascade inflammatory response[20].

Mitochondria-derived ROS is the key stimuli to trigger the activation of NLRP3 inflammasome[21]. When the levels of oxygen fall, hypoxia would damage mitochondria and induce ROS production[22-24]. Mitophagy can eliminate damaged mitochondria, reduce ROS production, and alleviate NLRP3 inflammasome activation[25]. It remains to be ascertained whether hypercapnia would activate the NLRP3 inflammasome through inhibiting mitophagy in hypoxia-activated microglia.

In the present study, we hypothesized that hypercapnia may promote microglial pyroptosis via inhibiting mitophagy in adult rats with hypoxemia. It was surmised that hypercapnia might exert its effect through inhibiting mitophagy, inducing ROS production, activating NLRP3 inflammasome, which ultimately would lead to IL-18 and IL-1 $\beta$ maturation and pyroptosis activation.

\section{Methods}

\section{Animals and experimental groups}

Male adult Sprague-Dawley (SD) rats $(n=128)$ aged about 3 months and weighing approximately 220 to $250 \mathrm{~g}$ were randomly divided into five groups: sham-operated group (abbreviated Sham group), Hypercapnia group, Hypoxemia group, Hypercapnia + Hypoxemia group (abbreviated HH group), Hypercapnia + Hypoxemia + N-Acetyl-L-cysteine group (abbreviated HH + NAC group).

\section{The rat model of hypercapnia/hypoxemia}

All rats were fasted with access to water overnight before the experiments. The rat model of hypercapnia/hypoxemia was established as described in our previous study[9]. Briefly, the rats were anesthetized with pentobarbital sodium followed by mechanical ventilation. The tidal volume $(9 \mathrm{ml} / \mathrm{kg}$ body weight), respiratory rate (45 breaths/min), and inspiratory to expiratory ratio (1:1) were fixed. Sham group was exposed to the air. Hypercapnia group was exposed to $5 \% \mathrm{CO}_{2}$ to maintain the $\mathrm{pH}$ of arterial blood at 7.20 - 7.25. Hypoxemia group was exposed to $16 \% \mathrm{O}_{2}$ to maintain partial pressure of arterial blood oxygen $\left(\mathrm{PaO}_{2}\right)$ at $55-60 \mathrm{mmHg}$. $\mathrm{HH}$ group was exposed to $16 \% \mathrm{O}_{2}$ mixing with $5 \% \mathrm{CO}_{2}$ to maintain $\mathrm{PaO}_{2}$ at $55-60 \mathrm{mmHg}$ and $\mathrm{pH}$ at $7.20-7.25$. Rats in the $\mathrm{HH}+\mathrm{NAC}$ group were treated with an intraperitoneal injection of $150 \mathrm{mg} / \mathrm{kg} \mathrm{NAC}$ (MedChemExpress, Monmouth, NJ, USA; cat. no. HY-B0215) for 30 min before being exposed to $5 \% \mathrm{CO}_{2}$ and $16 \% \mathrm{O}_{2}$. The rats, which were used for Western blotting analysis and immunofluorescence staining, were not performed invasive manipulation except ventilation. 


\section{Measurement of cerebral oxygen extraction ratio $\left(\mathrm{CERO}_{2}\right)$}

The right femoral artery and the right jugular vein was cannulated upstream. The blood samples were collected at $3 \mathrm{~h}$ after ventilation. The hemoglobin concentration $(\mathrm{Hb})$, saturation of arterial blood oxygen $\left(\mathrm{SaO}_{2}\right), \mathrm{PaO}_{2}$, saturation of jugular venous blood oxygen $\left(\mathrm{SjVO}_{2}\right)$, and pressure of jugular venous blood oxygen $\left(\mathrm{PjVO}_{2}\right.$ ) were measured using a blood gas/electrolyte analyzer (Model 5700, IL, San Diego, CA, USA). The content of arterial blood oxygen $\left(\mathrm{CaO}_{2}\right)$, content of jugular venous blood oxygen $\left(\mathrm{CjVO}_{2}\right)$, and $\mathrm{CERO}_{2}$ were calculated using the following formulas:

\section{See Formulas 1, 2, and 3 in the Supplemental Files section.}

\section{Measurement of the partial pressure of brain tissue oxygen $\left(\mathrm{PbtO}_{2}\right)$}

The levels of $\mathrm{PbtO}_{2}$ were measured at $0.5,1,1.5,2,2.5$, and $3 \mathrm{~h}$ after ventilation. To evaluate the $\mathrm{PbtO}_{2}$, a midline incision over vertex was performed after anesthesia. After this, a hole was drilled caudal to the coronal suture, $4 \mathrm{~mm}$ from the midline. The dura was punctured and a microsensor for $\mathrm{PbtO}_{2}$ was inserted into brain tissue[26]. A monitor (Integra CAMO2, Integra LifeSciences Limited, County Offaly, Ireland) was used to measure the $\mathrm{PbtO}_{2}$.

\section{ROS evaluation of brain tissue}

The ROS of brain tissue was evaluated using a ROS ELISA kit (Dogesce, Beijing, China; cat. no. DG21175D) at $3 \mathrm{~h}$ after ventilation following the manufacturers' instructions. Briefly, samples and standards $(50 \mu \mathrm{l} /$ well) were added to the plate wells coated by antibodies labeled with HRP, which were used to capture ROS. The plate was incubated for $1 \mathrm{~h}$ at $37^{\circ} \mathrm{C}$. After washing completely, substrate A (50 $\mu \mathrm{l} /$ well) and substrate $\mathrm{B}\left(50 \mu \mathrm{l} /\right.$ well) were added to incubate the plate in a dark place for $15 \mathrm{~min}$ at $37^{\circ} \mathrm{C}$. Then the stop buffer was added, and the optical density (OD) was measured spectrophotometrically at a wavelength of $450 \mathrm{~nm}$. The concentrations of ROS in the samples were then determined by comparing the optical density of the samples to the standard curve.

\section{Transmission Electron Microscopy}

Transmission Electron Microscopy (TEM) measurements were performed using an $\mathrm{H}-7500$ transmission electron microscope (Hitachi, Chiyoda, Tokyo). Rats were anesthetized with pentobarbital sodium (30 $\mathrm{mg} / \mathrm{kg}$ intraperitoneal injection) followed by the perfusion of $2.5 \%$ glutaraldehyde. The hippocampus was cut into $1 \mathrm{~mm}^{3}$ and fixed in $1 \%$ osmium tetroxide. The tissue samples were then dehydrated in graded ethyl alcohol and embedded in epoxy resin. Ultrathin sections were cut and stained in uranyl acetate and lead citrate. The sections were examined under the electron microscope.

BV-2 microglial cell cultures and treatment 
BV-2 microglial cells were purchased form CHI Scientific (cat. no. 7-1502), and were cultured and treated as described in our previous study[9]. Briefly, the cells were cultured with DMEM high glucose (Invitrogen Life Technologies Corporation, Carlsbad, CA, USA; cat. no. 8117121) supplemented with $10 \%$ FBS (Invitrogen Life Technologies Corporation; Carlsbad, CA, USA; cat. no. 42F0374K). The microglial cells were randomly divided into five groups: Control group, high concentration of carbon dioxide group (abbreviated $\mathrm{HC}$ group), Hypoxia group, Hypoxia $+\mathrm{HC}$ group, and Hypoxia + HC + NAC group. Control group was exposed to $5 \% \mathrm{CO}_{2}+20 \% \mathrm{O}_{2}$. $\mathrm{HC}$ group was exposed to $15 \% \mathrm{CO}_{2}+20 \% \mathrm{O}_{2}$ to maintain the $\mathrm{pH}$ of the supernatant at $7.20-7.25$. Hypoxia group was exposed to $5 \% \mathrm{CO}_{2}+0.2 \% \mathrm{O}_{2}$ to maintain partial pressure of oxygen $\left(\mathrm{PO}_{2}\right)$ of the supernatant at $55-60 \mathrm{mmHg}$. Hypoxia $+\mathrm{HC}$ group was exposed to $15 \%$ $\mathrm{CO}_{2}+0.2 \% \mathrm{O}_{2}$ to maintain $\mathrm{PO}_{2}$ at $55-60 \mathrm{mmHg}$ and $\mathrm{pH}$ at $7.20-7.25$. The cells in the Hypoxia $+\mathrm{HC}+$ NAC group were treated with 2 mM NAC (MedChemExpress, Monmouth, NJ, USA; cat. no. HY-B0215) for $30 \mathrm{~min}$ before being exposed to $15 \% \mathrm{CO}_{2}+0.2 \% \mathrm{O}_{2}$.

\section{Oxygen consumption rate (OCR) evaluation of BV-2 microglial cells}

The OCR was evaluated using a cellulate OCR Assay Kit (BestBio, Shanghai, China; cat. no. BB-48211) after treatment with $0.2 \% \mathrm{O}_{2}$ and $15 \% \mathrm{CO}_{2}$ for $0 \mathrm{~h}, 6 \mathrm{~h}, 12 \mathrm{~h}$, and $24 \mathrm{~h}$. The intervention time and testing time were chosen, when the levels of OCR peaked (In this study, $12 \mathrm{~h}$ was chosen as the intervention time, and 18 min was chosen as the testing time) (Fig. 1C). The OCR was measured following the manufacturers' instructions. Briefly, BV-2 microglial cells were seeded in $96-$ well plates ( 5000 cells/well) and cultured in DMEM high glucose supplemented with $10 \%$ FBS. When the cells spread to $80 \%$ of the bottom of the well, they were treated with different concentrations of $\mathrm{CO}_{2}$ and $\mathrm{O}_{2}$. After the treatment, the medium was changed to DMEM high glucose without FBS, and then fluorescent probes $(10 \mu \mathrm{l} /$ well) were added sequentially. Finally, an oxygen mounting medium was added ( 2 drops/well). The OCR levels were examined every three minutes until half an hour with a fluorescent microplate reader (Model 9260, IL$\mathrm{COR}^{\circledR}$ inc, LINCOLN, NE, USA). The excited and emitted wavelengths were $485 / 20 \mathrm{~nm}$ and 590/35 nm, respectively.

\section{ROS measurement in microglia}

The ROS production in BV-2 microglial cells was evaluated using a ROS assay kit (BestBio, Shanghai, China; cat. no. BB-4705-2) following the manufacturers' instructions. Briefly, DCFH-DA was diluted with DMEM high glucose without FBS (1: 1500). The coverslips with adherent BV-2 microglial cells were cultured with DMEM high glucose supplemented with $10 \%$ FBS. After the treatment, the medium was changed to diluted DCFH-DA ( $2 \mathrm{ml} /$ well). Then the plates were incubated for $20 \mathrm{~min}$ at $37{ }^{\circ} \mathrm{C}, 5 \% \mathrm{CO}_{2}$. The coverslips were washed with DMEM high glucose without FBS. Finally, the coverslips were mounted by a fluorescent mounting medium and detected using a fluorescence microscope (Olympus DP73 Microscope, Olympus, Tokyo, Japan).

\section{Western blotting analysis.}


Total proteins from the hippocampus tissue and BV-2 microglial cells ( $n=4$ for each group) were extracted using a Total Protein Extraction Kit (BestBio, Shanghai, China; cat. no. BB-3101-100T). Protein concentrations were determined using a BCA Protein Assay Kit (Invitrogen Life Technologies Corporation, Carlsbad, CA, USA; cat. no. 23227). Equal amounts of protein from each sample were separated in a $15 \%$ SDS-PAGE gel and transferred to PVDF membranes, which were blocked with $5 \%$ non-fat milk for $1 \mathrm{~h}$ at room temperature. After this, the following primary antibodies were added to incubate the membranes overnight at $4{ }^{\circ} \mathrm{C}$ : LC3B antibody (1: 1000, Abcam, Cambridge, MA, USA; cat. no. ab48394), SQSTM1/p62 (1: 1000, Abcam, Cambridge, MA, USA; cat. no. ab56416), Gasdermin D (1: 1000, Cell Signaling Technology; cat. no. 93709), caspase-1 (1: 1000, Abcam, Cambridge, MA, USA; cat. no. ab1872), IL-18 (1: 1000, Abcam, Cambridge, MA, USA; Cat. No. ab207323) and IL-1 $\beta$ (1: 1000, Abcam, Cambridge, MA, USA; Cat. No. ab9722). The membranes were washed on the following day, and the HRP-labeled goat antirabbit antibody (1: 3000; Cell Signaling Technology; cat. no. 7074S) was added to incubate the membranes for $2 \mathrm{~h}$ at $4{ }^{\circ} \mathrm{C}$. The immunoblots were visualized using a chemiluminescence kit (Bioworld Technology, St. Louis Park, MN, USA; cat. no. AC36131), and detected by an imaging densitometer (ImageQuant LAS 500, GE Healthcare Bio-Sciences AB, Uppsala, Sweden). The relative density was quantified using FluorChem 8900 software (version 4.0.1, Alpha Innotech Corporation, San Leandro CA, USA). $\beta$-actin was used as the control.

\section{Double immunofluorescence labeling}

In vivo, the rats were anesthetized with pentobarbital sodium and transcardially perfused with normal saline and $4 \%$ paraformaldehyde at $3 \mathrm{~h}$ after ventilation. The brains were harvested and post-fixed in $4 \%$ paraformaldehyde. This tissue was then dehydrated in graded sucrose and cut into sections of $10 \mu \mathrm{m}$ thickness. The sections were blocked in $5 \%$ normal donkey serum for $0.5 \mathrm{~h}$ at room temperature. In vitro, the coverslips with adherent BV-2 microglial cells were fixed with $4 \%$ paraformaldehyde at $24 \mathrm{~h}$ after treatment. The coverslips were blocked in $5 \%$ normal donkey serum for $0.5 \mathrm{~h}$ at room temperature sequentially.

After that, the following primary antibodies were added to incubate the sections/coverslips overnight at 4 'C: caspase-1 (1: 100; Abcam, Cambridge, MA, USA; cat. no. ab1872), IL-1ß (1: 100; Abcam, Cambridge, MA, USA; cat. no. ab9722), IL-18 (1: 100, Abcam, Cambridge, MA, USA; Cat. No. ab207323), and Iba1 (1: 100; Abcam, Cambridge, MA, USA; cat. no. ab15690). The sections/coverslips were washed on the following day, and the secondary antibodies Alexa Fluor ${ }^{\circledR} 549$ Goat Anti-Rabbit lgG $(H+L)$ (1:100; Invitrogen Life Technologies, Carlsbad, CA, USA; cat. no. ATRJN1301) and Alexa Fluor ${ }^{\circledR} 488$ Goat antimouse IgG (1:100; Invitrogen Life Technologies, Carlsbad, CA, USA; cat. no. ATRMR2301) were added to incubate the sections/coverslips for $1 \mathrm{~h}$ at room temperature. Finally, the sections/coverslips were mounted by the fluorescent mounting medium with DAPI (Sigma, St. Louis, MO, USA; cat. no. SLBW4468) and detected using a fluorescence microscope (Olympus DP73 Microscope, Olympus, Tokyo, Japan).

\section{Statistical analysis}


The statistical analysis was performed by the SPSS19.0 statistical (IBM, New York, USA). All values are expressed as mean \pm standard deviation. Repeated measures one-way analysis of variance (ANOVA) was used to analyze the repeated measurement data. Factorial ANOVA was for the interaction effects. When an interaction was examined, simple effects analyses were evaluated. Differences were considered statistically significant if the $P$-value $<0.05$.

\section{Results}

\section{Hypercapnia increased cerebral oxygen extraction rate $\left(\mathrm{CERO}_{2}\right)$ in hypoxemic rats}

Significant interaction effects were observed between hypercapnia treatment and hypoxia treatment $(P<$ 0.01) (Fig. 1A). Simple effects analyses found an increased $\mathrm{CERO}_{2}$ in Hypoxemia group $(P<0.05)$, but not in Hypercapnia group $(P>0.05)$ compared with Sham group. $\mathrm{HH}$ group had the highest $\mathrm{CERO}_{2}$ levels as compared with Hypoxemia group $(P<0.01)$ and Hypercapnia group $(P<0.01)$ (Fig. 1B).

\section{$15 \% \mathrm{CO}_{2}$ increased the oxygen consumption rate (OCR) in hypoxic BV-2 microglial cells}

The OCR levels of BV-2 microglial cells were examined after the treatment with $0.2 \% \mathrm{O}_{2}$ and $15 \% \mathrm{CO}_{2}$ for $0 \mathrm{~h}, 6 \mathrm{~h}, 12 \mathrm{~h}$, and $24 \mathrm{~h}$. The OCR levels were the highest in $12 \mathrm{~h}$ group as compared with other groups (12 h vs 0 h: $P<0.01 ; 12$ h vs 6 h: $P>0.05 ; 12$ h vs 24 h: $P<0.01)$. Besides, the OCR levels were increased time-dependently and achieved a stationary phase at $18 \mathrm{~min}$ (Fig. 1C). Because of this, microglia were treated for 12 hours, and the OCR was examined at 18 min after the treatment (Fig. 1D, E). Significant interaction effects were observed between $0.2 \% \mathrm{O}_{2}$ treatment and $15 \% \mathrm{CO}_{2}$ treatment $(P<0.01)$ (Fig. 1D). Simple effects analyses found increased levels of OCR in Hypoxia group $(P<0.01)$, but not in HC group $(P>0.05)$ compared with Control group. Hypoxia $+\mathrm{HC}$ group had the highest levels of OCR as compared with Hypoxia group $(P<0.01)$ and $\mathrm{HC}$ group $(P<0.01)$ (Fig. 1E).

\section{Hypercapnia decreased partial pressure of brain tissue oxygen $\left(\mathrm{PbtO}_{2}\right)$ in hypoxemic rats}

Compared with the Sham group, decreased $\mathrm{PbtO}_{2}$ levels were found in Hypoxemia group $(0.5 \mathrm{~h}-3 \mathrm{~h}: P<$ 0.01 ), but not in Hypercapnia group ( $0.5 \mathrm{~h}-3 \mathrm{~h}: P>0.05$ ) (Fig. $2 \mathrm{~A}-\mathrm{C}$ ). $\mathrm{HH}$ group had higher $\mathrm{PbtO}_{2}$ levels as compared with Hypoxemia group in the first 1.5 hours $(0.5 \mathrm{~h}: P<0.01 ; 1 \mathrm{~h}: P<0.01 ; 1.5 \mathrm{~h}: P<0.05)$ (Fig. 2B). On the contrary, $\mathrm{HH}$ group had lower $\mathrm{PbtO}_{2}$ levels as compared with Hypoxemia group after 2 hours (2.5 h: $P<0.05 ; 3$ h: $P<0.05)$ (Fig. 2 C).

\section{Hypercapnia inhibited mitophagy in microglia in the hypoxic hippocampus}

Significant interaction effects were observed between hypercapnia treatment and hypoxia treatment (LC3II: $P<0.01$, p62: $P<0.01$ ) (Fig. 3B, C). Simple effects analyses found increased protein expression levels of LC3-II in Hypoxemia group compared with Sham group $(P<0.01)$. HH group showed lower expression levels of LC3-II in comparison with Hypoxemia group $(P<0.01)$ (Fig. 3D). In contrast, a significant decrease in p62 expression was observed in Hypoxemia group compared with Sham group $(P<0.01)$. 
The levels of p62 expression in HH group were higher compared with Hypoxemia group $(P<0.01)$ (Fig. $3 \mathrm{E})$. Transmission electron microscope images of microglia in the hypoxic hippocampus showed ultrastructural alterations with hypercapnia treatment (Fig. 3F). The mitochondria exhibited normal morphology in sham group (Fig. 3F a, e) and hypercapnia group(Fig. 3F b, f). The most prominent morphologic change in hypoxic microglia was the formation of abundant autophagic vacuoles enveloping mitochondria (Fig. $3 \mathrm{~F} \mathrm{c}, \mathrm{g}$ ). However, the autophagic vacuoles were rarely observed in hypercapnia + hypoxia treated microglia (Fig. 3F d, h).

\section{$15 \% \mathrm{CO}_{2}$ inhibited mitophagy in hypoxia-activated BV-2 microglia}

Significant interaction effects were observed between $0.2 \% \mathrm{O}_{2}$ treatment and $15 \% \mathrm{CO}_{2}$ treatment (LC3-II: $P<0.01$, p62: $P<0.01$ ) (Fig. 4B, C). Simple effects analyses found increased protein expression levels of LC3-II in Hypoxia group $(P<0.01)$ compared with Control group. Hypoxia + HC group showed lower expression levels of LC3-II in comparison with Hypoxia group $(P<0.01)$ (Fig. 4D). In contrast, a significant decrease in p62 expression was observed in Hypoxia group compared with Control group $(P<0.01)$. The levels of p62 expression in Hypoxia + HC group were higher compared with Hypoxia group $(P<0.01)$ (Fig. $4 \mathrm{E})$.

\section{Hypercapnia induced overproduction of ROS in the hypoxemic hippocampus}

Significant interaction effects were observed between hypercapnia treatment and hypoxia treatment $(P<$ 0.05) (Fig. 5A). Simple effects analyses found increased levels of ROS in Hypoxemia group $(P<0.01)$, but not in Hypercapnia group $(P>0.05)$ compared with Sham group. $\mathrm{HH}$ group had the highest ROS levels as compared with Hypoxemia group $(P<0.01)$ and Hypercapnia group $(P<0.01)$ (Fig. 5B).

\section{$15 \% \mathrm{CO}_{2}$ induced overproduction of ROS in hypoxic BV-2 microglial cells}

Enhanced ROS immunofluorescence was observed in Hypoxia group, but not in $\mathrm{HC}$ group compared with Control group. Hypoxia + HC group had the strongest ROS fluorescence as compared with Hypoxia group and $\mathrm{HC}$ group. ROS fluorescence was obviously reduced with NAC pretreatment (2 mM) in BV-2 microglial cells (Fig. 5C).

\section{Hypercapnia enhanced NLRP3 inflammasome activation via inducing ROS overproduction in microglia in the hypoxic hippocampus}

Significant interaction effects were observed between hypercapnia treatment and hypoxia treatment $(P<$ 0.01 ) (Fig. 6B). Simple effects analyses found increased protein expression levels of caspase- 1 in Hypoxemia group $(P<0.01)$, but not in Hypercapnia group $(P>0.05)$ compared with Sham group. $\mathrm{HH}$ group had the highest expression levels of caspase- 1 as compared with Hypoxemia group $(P<0.01)$ and Hypercapnia group $(P<0.01)$ (Fig. $6 \mathrm{C})$. Additionally, the protein expression of caspase-1 was significantly suppressed with NAC pretreatment $(150 \mathrm{mg} / \mathrm{kg})$ in rats $(P<0.01)$ (Fig. 6C). Double immunofluorescence was used to examine caspase-1 expression in microglia of hippocampus (Fig. 6D). Enhanced caspase-1 
immunofluorescence was observed in Hypoxemia group, but not in Hypercapnia group compared with Sham group. $\mathrm{HH}$ group had the most intense caspase-1 fluorescence when compared with Hypoxemia group and Hypercapnia group. In rats given NAC treatment, caspase-1 fluorescence was noticeably attenuated (Fig. 6D).

$15 \% \mathrm{CO}_{2}$ enhanced NLRP3 inflammasome activation via inducing ROS overproduction in hypoxic BV-2 microglial cells

Significant interaction effects were observed between $0.2 \% \mathrm{O}_{2}$ treatment and $15 \% \mathrm{CO}_{2}$ treatment $(P<$ 0.01 ) (Fig. 7B). Simple effects analyses found increased protein expression levels of caspase-1 in Hypoxia group $(P<0.01)$, but not in $\mathrm{HC}$ group $(P>0.05)$ compared with Control group. Hypoxia $+\mathrm{HC}$ group had the highest expression levels of caspase-1 as compared with Hypoxia group $(P<0.01)$ and $\mathrm{HC}$ group $(P<0.01)$ (Fig. 7C). Additionally, the protein expression of caspase-1 was significantly suppressed with NAC pretreatment $(2 \mathrm{mM})$ in BV-2 microglial cells $(P<0.01)$ (Fig. 7C). Double immunofluorescence was used to examine caspase-1 expression in BV-2 microglial cells (Fig. 7D). Enhanced caspase-1 immunofluorescence was observed in Hypoxia group, but not in $\mathrm{HC}$ group compared with Control group. Hypoxia $+\mathrm{HC}$ group had the strongest caspase-1 fluorescence as compared with Hypoxia group and HC group. Of note, caspase- 1 fluorescence was evidently reduced with NAC pretreatment (2 mM) in BV-2 microglial cells (Fig. 7D).

Hypercapnia increased GSDMD-N expression via inducing ROS overproduction in microglia in the hypoxic hippocampus

Significant interaction effects were observed between hypercapnia treatment and hypoxia treatment $(P<$ 0.01) (Fig. 8B). Simple effects analyses found increased protein expression levels of GSDMD-N in Hypoxemia group $(P<0.05)$, but not in Hypercapnia group $(P>0.05)$ compared with Sham group. $\mathrm{HH}$ group showed the highest expression levels of GSDMD-N in comparison with Hypoxemia group $(P<0.01)$ and Hypercapnia group $(P<0.01)$ (Fig. 8C). Additionally, the protein expression of GSDMD-N was significantly suppressed with NAC pretreatment $(150 \mathrm{mg} / \mathrm{kg})$ in rats $(P<0.01)$ (Fig. 8C).

\section{$15 \% \mathrm{CO}_{2}$ increased GSDMD-N expression via inducing ROS overproduction in hypoxic BV-2 microglial cells}

Significant interaction effects were observed between $0.2 \% \mathrm{O}_{2}$ treatment and $15 \% \mathrm{CO}_{2}$ treatment $(P<$ 0.01) (Fig. 8E). Simple effects analyses found increased protein expression levels of GSDMD-N in Hypoxia group $(P<0.01)$, but not in $\mathrm{HC}$ group $(P>0.05)$ compared with Control group. Hypoxia $+\mathrm{HC}$ group had the highest expression levels of GSDMD-N as compared with Hypoxia group $(P<0.01)$ and $\mathrm{HC}$ group $(P<0.01)$ (Fig. 8F). Additionally, the protein expression of GSDMD-N was significantly suppressed with NAC pretreatment $(2 \mathrm{mM})$ in BV-2 microglial cells $(P<0.01)$ (Fig. 8F).

Hypercapnia increased IL-1 $\beta$ expression via inducing ROS overproduction in microglia in the hypoxic hippocampus 
Significant interaction effects were observed between hypercapnia treatment and hypoxia treatment $(P<$ 0.01) (Fig. 9B). Simple effects analyses found increased protein expression levels of IL-1 $\beta$ in Hypoxemia group $(P<0.01)$, but not in Hypercapnia group $(P>0.05)$ compared with Sham group. HH group showed the highest expression levels of IL-1 $\beta$ in comparison with Hypoxemia group $(P<0.01)$ and Hypercapnia group $(P<0.01)$ (Fig. 9C). Additionally, the protein expression of IL-1 $\beta$ was significantly suppressed with NAC pretreatment $(150 \mathrm{mg} / \mathrm{kg})$ in rats $(P<0.01)$ (Fig. $9 \mathrm{C})$. Double immunofluorescence was used to examine IL-1 $\beta$ expression in microglia of hippocampus (Fig. 9D). Enhanced IL-1 $\beta$ immunofluorescence was observed in Hypoxemia group, but not in Hypercapnia group compared with Sham group. $\mathrm{HH}$ group had the strongest IL-1 $\beta$ fluorescence as compared with Hypoxemia group and Hypercapnia group. IL-1 $\beta$ fluorescence was evidently reduced in rats given NAC pretreatment (Fig. 9D).

\section{$15 \% \mathrm{CO}_{2}$ increased IL-1 $\beta$ expression via inducing ROS overproduction in hypoxic BV-2 microglial cells}

Significant interaction effects were observed between $0.2 \% \mathrm{O}_{2}$ treatment and $15 \% \mathrm{CO}_{2}$ treatment $(P<$ 0.01) (Fig. 10B). Simple effects analyses found increased protein expression levels of IL-1 $\beta$ in Hypoxia group $(P<0.01)$, but not in $\mathrm{HC}$ group $(P>0.05)$ compared with Control group. Hypoxia $+\mathrm{HC}$ group had the highest expression levels of IL-1 $\beta$ as compared with Hypoxia group $(P<0.01)$ and $\mathrm{HC}$ group $(P<0.01)$ (Fig. 10C). Additionally, the protein expression of IL-1 $\beta$ was significantly suppressed with NAC pretreatment $(2 \mathrm{mM})$ in BV-2 microglial cells $(P<0.01)$ (Fig. 10C). Double immunofluorescence confirmed IL-1 $\beta$ expression in BV-2 microglial cells (Fig. 10D). Enhanced IL-1 $\beta$ immunofluorescence was observed in Hypoxia group, but not in $\mathrm{HC}$ group compared with Control group. Hypoxia $+\mathrm{HC}$ group had the strongest IL-1 $\beta$ fluorescence as compared with Hypoxia group and HC group. IL-1 $\beta$ fluorescence was markedly suppressed with NAC pretreatment (2 mM) in BV-2 microglial cells (Fig. 10D).

\section{Hypercapnia increased IL-18 expression via inducing ROS overproduction in microglia in the hypoxic hippocampus}

Significant interaction effects were observed between hypercapnia treatment and hypoxia treatment $(P<$ 0.01) (Fig. 11B). Simple effects analyses found increased protein expression levels of IL-18 in Hypoxemia group $(P<0.01)$, but not in Hypercapnia group $(P>0.05)$ compared with Sham group. HH group showed the highest expression levels of IL-18 in comparison with Hypoxemia group $(P<0.01)$ and Hypercapnia group $(P<0.01)$ (Fig. 11C). Additionally, the protein expression of IL-18 was significantly suppressed with NAC pretreatment $(150 \mathrm{mg} / \mathrm{kg})$ in rats $(P<0.01)$ (Fig. 11C). Double immunofluorescence was used to examine IL-18 expression in microglia of hippocampus (Fig. 11D). Enhanced IL-18 immunofluorescence was observed in Hypoxemia group, but not in Hypercapnia group compared with Sham group. HH group had the strongest IL-18 fluorescence as compared with Hypoxemia group and Hypercapnia group. IL-18 fluorescence was evidently reduced in rats given NAC pretreatment (Fig. 11D).

\section{$15 \% \mathrm{CO}_{2}$ increased IL-18 expression via inducing ROS overproduction in hypoxic BV-2 microglial cells}

Significant interaction effects were observed between $0.2 \% \mathrm{O}_{2}$ treatment and $15 \% \mathrm{CO}_{2}$ treatment $(P<$ 0.01) (Fig. 12B). Simple effects analyses found increased protein expression levels of IL-18 in Hypoxia 
group $(P<0.05)$, but not in $\mathrm{HC}$ group $(P>0.05)$ compared with Control group. Hypoxia $+\mathrm{HC}$ group had the highest expression levels of IL-18 as compared with Hypoxia group $(P<0.01)$ and $\mathrm{HC}$ group $(P<0.01)$ (Fig. 12C). Additionally, the protein expression of IL-18 was significantly suppressed with NAC pretreatment $(2 \mathrm{mM})$ in BV-2 microglial cells $(P<0.01)$ (Fig. 12C). Double immunofluorescence confirmed IL-18 expression in BV-2 microglial cells (Fig. 12D). Enhanced IL-18 immunofluorescence was observed in Hypoxia group, but not in $\mathrm{HC}$ group compared with Control group. Hypoxia + HC group had the strongest IL-18 fluorescence as compared with Hypoxia group and HC group. IL-18 fluorescence was markedly suppressed with NAC pretreatment (2 mM) in BV-2 microglial cells (Fig. 12D).

\section{Discussion}

The present results have shown that hypercapnia promoted pyroptosis via inhibiting mitophagy in hypoxia-activated microglia in vitro and in vivo. This was evident by the increased $\mathrm{CERO}_{2}$, decreased $\mathrm{PbtO}_{2}$, and increased expression levels of LC3-II, caspase-1, GSDMD-N, IL-18, and IL-1 $\beta$ in hypoxiaactivated microglia cells.

The present rat and cell models of hypercapnia/hypoxemia were established as described in our previous study[9]. In the models, $\mathrm{PO}_{2}$ levels of the arterial blood and culture supernatant were maintained at 55$60 \mathrm{mmHg}$ and $\mathrm{pH}$ levels of the arterial blood and culture supernatant at 7.20-7.25. These are consistent with the change of hypoxemia and permissive hypercapnia in ARDS[27-30].

We reported in our previous study that hypercapnia could induce IL-1 $\beta$ overproduction in hypoxiaactivated microglia. However, the underlying mechanism has remained unclear. It has been reported that the expression of caspase- 1 would be upregulated after NLRP3 inflammasome being activated. Caspase1 could lead to IL-18 and IL-1 $\beta$ maturation and pyroptosis activation. GSDMD-N is the executioner of pyroptosis. GSDMD-N helped to form a pore in the plasma membrane, from that IL- $1 \beta$ and IL-18 are released $[19,20]$. Under hypoxic conditions, ROS is known to be critical for NLRP3 inflammasome activation[31-33]. Thus, the expression levels of caspase-1, GSDMD-N, IL-18, and IL-1 $\beta$ in the microglia in this study were determined, and the production of ROS was also evaluated both in vivo and in vitro. Significant interaction effects were observed on ROS production, caspase-1, GSDMD-N, IL-18, and IL-1 $\beta$ expression between hypercapnia treatment and hypoxia treatment. More importantly, we have shown that hypercapnia upregulated the expression of caspase-1, GSDMD-N, IL-18, and IL-1 $\beta$ in hypoxic microglia via inducing ROS overproduction. Remarkably, caspase-1, GSDMD-N, IL-18, and IL-1 $\beta$ expression in hypoxic microglia was downregulated when ROS was scavenged by NAC. These results indicate that hypercapnia promotes microglial pyroptosis via inducing ROS overproduction and activating the NLRP3 inflammasome.

When the levels of oxygen fall, hypoxia would damage mitochondria and induce ROS production[22-24]. Mitophagy can eliminate damaged mitochondria, reduce ROS production, and then alleviate NLRP3 inflammasome activation[25]. To ascertain if hypercapnia would activate the NLRP3 inflammasome through inhibiting mitophagy in hypoxia-activated microglia, expression of LC3-II and p62 in the microglia 
was determined. And mitophagy was observed using transmission electron microscopy (TEM). We found that the expression levels of LC3-II were reduced, while those of p62 were increased by hypercapnia in the hypoxic hippocampus and BV-2 microglia. Autophagosomes could be seen in hypoxia treated microglial cells under TEM, while those were rarely observed in hypercapnia + hypoxia treated microglia. These results indicate that hypercapnia might enhance the NLRP3 inflammasome activation in hypoxiaactivated microglial cells via inhibiting mitophagy.

To determine the effect of hypercapnia on oxidative metabolism of the rat brain, cerebral oxygen extraction ratio $\left(\mathrm{CERO}_{2}\right)$ and partial pressure of brain tissue oxygen $\left(\mathrm{PbtO}_{2}\right)$ were examined. Hypoxemia, as a typical symptom of ARDS, decreases the oxidative metabolism rate of the brain to avoid or reduce cerebral damage via preventing the $\mathrm{PbtO}_{2}$ from dropping to a very low level[34]. In this study, we showed hypercapnia alone was not enough to increase or decrease the levels of $\mathrm{PbtO}_{2}$ and $\mathrm{CERO}_{2}$. In the first 1.5 hours, the levels of $\mathrm{PbtO}_{2}$ were elevated by hypercapnia in the hypoxemic rats. This may be the reason why hypercapnia was neuroprotective in rats with transient global cerebral ischemia-reperfusion injury[35] and lateral fluid percussion injury[36]. However, hypercapnia significantly decreased the levels of $\mathrm{PbtO}_{2}$ in the hypoxemic rats after 2 hours' ventilation. It was further evidenced by the increased levels of $\mathrm{CERO}_{2}$ at $3 \mathrm{~h}$ after ventilation. These results suggest that hypercapnia is neuroprotective within a very short period $(1.5 \mathrm{~h})$, but hypercapnia could aggravate neuronal functions injury over a protracted period via increasing $\mathrm{CERO}_{2}$ and decreasing $\mathrm{PbtO}_{2}$.

In vitro, to determine the effect of a high concentration of $\mathrm{CO}_{2}$ on oxidative metabolism in hypoxic BV-2 microglial cells, the oxygen consumption rate (OCR) was evaluated. There was an interaction effect on the levels of OCR between $0.2 \% \mathrm{O}_{2}$ treatment and $15 \% \mathrm{CO}_{2}$ treatment. The high concentration of $\mathrm{CO}_{2}$ treatment could increase the levels of OCR in hypoxic BV-2 microglial cells, which were consistent with that hypercapnia increased levels of $\mathrm{CERO}_{2}$ in hypoxic rats. The results suggest that hypercapnia could intensify microglial hypoxia both in vivo and in vitro.

In summary, the present results have demonstrated the underlying mechanism whereby hypercapnia can promote pyroptosis in hypoxic microglia. In this connection, hypercapnia was found to intensify the cerebral hypoxia via increasing $\mathrm{CERO}_{2}$ and decreasing $\mathrm{PbtO}_{2}$. ROS was overproduced by hypercapnia in hypoxic microglial cells coupled with mitophagy inhibition and activation of NLRP3 inflammasome. Remarkedly, caspase-1, GSDMD-N, IL-18, and IL-1 $\beta$ expression was downregulated when ROS was scavenged. Thus, hypercapnia-induced mitophagy inhibition, NLRP3 inflammasome activation, and pyroptosis in microglia may be a potential target to mitigate neuroinflammation.

\section{Conclusions}

Hypercapnia-induced mitophagy inhibition may promote pyroptosis and enhance IL-1 $\beta$ and IL-18 release in hypoxia-activated microglia (Fig. 13). 


\section{Declarations}

Acknowledgments

The authors would like to thank Mr. ZhengKang Ding and Mrs. ZiXi Yang for technical assistance.

\section{Funding}

This work was supported by the Natural Science Foundation of Guangdong province (2016A030311043 and 2017A030313691) and the Medical Scientific Research Foundation of Guangdong Province (A2019135).

\section{Availability of data and materials}

The datasets used and analyzed during the current study are available from the corresponding author on reasonable request.

\section{Authors' contributions}

DHG participated in making the animal model, assessed IL-1 $\beta$, GSDMD, and caspase- 1 expression in the hypoxic hippocampus microglia, collected data, and drafted the manuscript. LXS detected autophagy and measured cerebral oxygen extraction ratio and partial pressure of brain tissue oxygen. LXQ conducted BV-2 microglial cells culture and treatment and observed mitophagy using transmission electron microscopy. WKR participated in evaluating ROS production of brain tissue and BV-2 microglial cells. LY participated in the evaluation of oxygen consumption rate evaluation in vitro. WMY performed the statistical analysis. ZHK carried out the design of the study. All authors read and approved the final manuscript.

\section{Ethics approval}

The Experimental Animal Care and Use Committee at Jinan University approved all animal procedure protocols (No. 20171011001), which conform to the Guide for the Care and Use of Laboratory Animals published by the US National Institutes of Health. All efforts were made to minimize the numbers of animals used and ensure minimal suffering.

\section{Consent for publication}

Not applicable

\section{Competing interests}

The authors declare that they have no competing interests.

\section{Author Details}


${ }^{1}$ Department of Emergency and Critical Care Medicine, Guangdong Provincial People's Hospital and Guangdong Academy of Medical Sciences, 106 ZhongshanEr Road, Guangzhou 510080, China.

${ }^{2}$ Southern Medical University, 1838 North Guangzhou Avenue, Guangzhou 510515, China. ${ }^{3}$ School of Medicine, South China University of Technology, Guangzhou Higher Education Mega Center, Guangzhou 510006, China

\section{Abbreviations}

ARDS: acute respiratory distress syndrome; CNS: central nervous system; IL-1 $\beta$ : interleukin-1 beta; NLRP3: NLR family, pyrin domain-containing 3; $\mathrm{PbtO}_{2}$ : partial pressure of brain tissue oxygen; OCR: oxygen consumption rate; ROS: reactive oxygen species; $\mathrm{Hb}$ : hemoglobin concentration; $\mathrm{PCO}_{2}$ : partial pressure of carbon dioxide; $\mathrm{PaO}_{2}$ : partial pressure of arterial blood oxygen; $\mathrm{SaO}_{2}$ : saturation of arterial blood oxygen; $\mathrm{PjVO}_{2}$ : pressure of jugular venous blood oxygen; $\mathrm{CaO}_{2}$ : content of arterial blood oxygen; $\mathrm{CjVO}_{2}$ : content of jugular venous blood oxygen; $\mathrm{SjVO}_{2}$ : saturation of jugular venous blood oxygen; GSDMD-FL: gasdermin D-full length; GSDMD-N: gasdermin D-N domains.

\section{References}

1. Papazian L, Aubron C, Brochard L, Chiche JD, Combes A, Dreyfuss D, et al. Formal guidelines: management of acute respiratory distress syndrome. Ann Intensive Care, 2019, 9: 69.

2. Matthay MA, Zemans RL, Zimmerman GA, Arabi YM, Beitler JR, Mercat A, et al. Acute respiratory distress syndrome. Nat Rev Dis Primers, 2019, 5: 18.

3. Duan EH, Adhikari N, D'Aragon F, Cook DJ, Mehta S, Alhazzani W, et al. Management of Acute Respiratory Distress Syndrome and Refractory Hypoxemia. A Multicenter Observational Study. Ann Am Thorac Soc, 2017, 14: 1818-1826.

4. Meade MO, Young D, Hanna S, Zhou Q, Bachman TE, Bollen C, et al. Severity of Hypoxemia and Effect of High-Frequency Oscillatory Ventilation in Acute Respiratory Distress Syndrome. Am J Respir Crit Care Med, 2017, 196: 727-733.

5. Shen Y, Cai G, Gong S, Dong L, Yan J, Cai W. Interaction between low tidal volume ventilation strategy and severity of acute respiratory distress syndrome: a retrospective cohort study. Crit Care, 2019, 23 : 254.

6. Fielding-Singh V, Matthay MA, Calfee CS. Beyond Low Tidal Volume Ventilation: Treatment Adjuncts for Severe Respiratory Failure in Acute Respiratory Distress Syndrome. Crit Care Med, 2018, 46: 18201831.

7. Brower RG, Matthay MA, Morris A, Schoenfeld D, Thompson BT, Wheeler A. Ventilation with lower tidal volumes as compared with traditional tidal volumes for acute lung injury and the acute respiratory distress syndrome. N Engl J Med, 2000, 342: 1301-1308.

8. Barnes T, Zochios V, Parhar K. Re-examining Permissive Hypercapnia in ARDS: A Narrative Review. Chest, 2018, 154(1): 185-195. 
9. Ding HG, Deng YY, Yang RQ, Wang QS, Jiang WQ, Han YL, et al. Hypercapnia induces IL-1 beta overproduction via activation of NLRP3 inflammasome: implication in cognitive impairment in hypoxemic adult rats. J Neuroinflammation, 2018, 15: 4.

10. Liu HD, Li W, Chen ZR, Hu YC, Zhang DD, Shen W, et al. Expression of the NLRP3 inflammasome in cerebral cortex after traumatic brain injury in a rat model. Neurochem Res, 2013, 38: 2072-2083.

11. Zhang N, Zhang X, Liu X, Wang H, Xue J, Yu J, et al. Chrysophanol inhibits NALP3 inflammasome activation and ameliorates cerebral ischemia/reperfusion in mice. Mediators Inflamm, 2014, 2014: 370530 .

12. Gustin A, Kirchmeyer M, Koncina E, Felten P, Losciuto S, Heurtaux T, et al. NLRP3 Inflammasome Is Expressed and Functional in Mouse Brain Microglia but Not in Astrocytes. PLoS One, 2015, 10 : e130624.

13. Hong P, Gu RN, Li FX, Xiong XX, Liang WB, You ZJ, et al. NLRP3 inflammasome as a potential treatment in ischemic stroke concomitant with diabetes. J Neuroinflammation, 2019, 16: 121.

14. Liu HJ, Pan XX, Liu BQ, Gui X, Hu L, Jiang CY, et al. Grape seed-derived procyanidins alleviate gout pain via NLRP3 inflammasome suppression. J Neuroinflammation, 2017, 14: 74.

15. Yue N, Huang $H$, Zhu X, Han Q, Wang Y, Li B, et al. Activation of P2X7 receptor and NLRP3 inflammasome assembly in hippocampal glial cells mediates chronic stress-induced depressive-like behaviors. J Neuroinflammation, 2017, 14: 102.

16. Kelley N, Jeltema D, Duan Y, He Y. The NLRP3 Inflammasome: An Overview of Mechanisms of Activation and Regulation. Int J Mol Sci, 2019, 20.

17. Hamilton C, Anand PK. Right place, right time: localisation and assembly of the NLRP3 inflammasome. F1000Res, 2019, 8.

18. Cookson BT, Brennan MA. Pro-inflammatory programmed cell death. Trends Microbiol, 2001, 9(3): 113-114.

19. Shi J, Zhao Y, Wang K, Shi X, Wang Y, Huang H, et al. Cleavage of GSDMD by inflammatory caspases determines pyroptotic cell death. Nature, 2015, 526(7575): 660-5

20. Ding J, Wang K, Liu W, She Y, Sun Q, Shi J, et al. Pore-forming activity and structural autoinhibition of the gasdermin family. Nature, 2016, 535(7610): 111-116.

21. Zhou R, Yazdi AS, Menu P, Tschopp J. A role for mitochondria in NLRP3 inflammasome activation. Nature, 2011, 469(7329): 221-5.

22. Murphy MP. How mitochondria produce reactive oxygen species. Biochem J, 2009, 417: 1-13.

23. Fuhrmann DC, Brune B. Mitochondrial composition and function under the control of hypoxia. Redox Biol, 2017, 12: 208-215.

24. Hernansanz-Agustin P, Ramos E, Navarro E, Parada E, Sanchez-Lopez N, Pelaez-Aguado L, et al. Mitochondrial complex I deactivation is related to superoxide production in acute hypoxia. Redox Biol, 2017, 12: 1040-1051. 
25. Deretic V, Levine B. Autophagy balances inflammation in innate immunity. Autophagy, 2018, 14(2): 243-251.

26. Wang CC, Kuo JR, Chen YC, Chio CC, Wang JJ, Lin BS. Brain tissue oxygen evaluation by wireless near-infrared spectroscopy. J Surg Res, 2016, 200: 669-675.

27. Nayak S, Jindal A. Permissive hypercapnia: Is there any upper limit? Indian J Crit Care Med, 2015, 19(1): 56-7.

28. Thorens JB, Jolliet P, Ritz M, Chevrolet JC. Effects of rapid permissive hypercapnia on hemodynamics, gas exchange, and oxygen transport and consumption during mechanical ventilation for the acute respiratory distress syndrome. Intensive Care Med, 1996, 22: 182-191.

29. Hickling KG, Walsh J, Henderson S, Jackson R. Low mortality rate in adult respiratory distress syndrome using low-volume, pressure-limited ventilation with permissive hypercapnia: a prospective study. Crit Care Med, 1994, 22: 1568-1578.

30. Dushianthan A, Cusack R, Chee N, Dunn JO, Grocott MP. Perceptions of diagnosis and management of patients with acute respiratory distress syndrome: a survey of United Kingdom intensive care physicians. BMC Anesthesiol, 2014, 14: 87.

31. Zeng J, Chen Y, Ding R, Feng L, Fu Z, Yang S, et al. Isoliquiritigenin alleviates early brain injury after experimental intracerebral hemorrhage via suppressing ROS- and/or NF-kappaB-mediated NLRP3 inflammasome activation by promoting Nrf2 antioxidant pathway. J Neuroinflammation, 2017, 14: 119.

32. Gong Z, Pan J, Shen Q, Li M, Peng Y. Mitochondrial dysfunction induces NLRP3 inflammasome activation during cerebral ischemia/reperfusion injury. J Neuroinflammation, 2018, 15: 242.

33. Tschopp J, Schroder K. NLRP3 inflammasome activation: The convergence of multiple signalling pathways on ROS production? Nat Rev Immunol, 2010, 10: 210-215.

34. Watts ME, Pocock R, Claudianos C. Brain Energy and Oxygen Metabolism: Emerging Role in Normal Function and Disease. Front Mol Neurosci, 2018, 11: 216.

35. Zhou Q, Cao B, Niu L, Cui X, Yu H, Liu J, et al. Effects of permissive hypercapnia on transient global cerebral ischemia-reperfusion injury in rats. Anesthesiology, 2010, 112: 288-297.

36. Yang WC, Wang Q, Chi LT, Wang YZ, Cao HL, Li WZ. Therapeutic hypercapnia reduces blood-brain barrier damage possibly via protein kinase Cepsilon in rats with lateral fluid percussion injury. $\mathrm{J}$ Neuroinflammation, 2019, 16: 36.

\section{Figures}



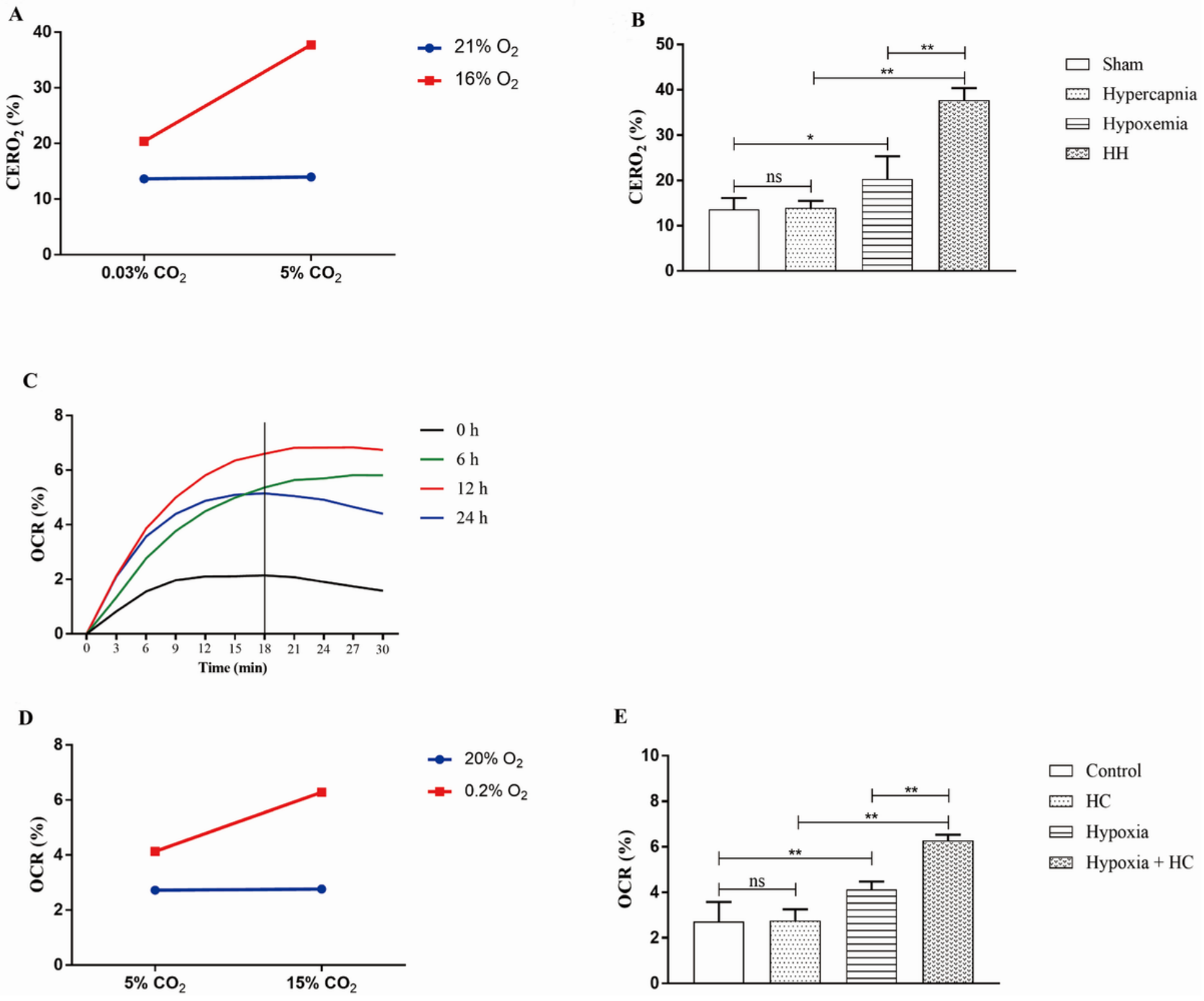

Figure 1

Hypercapnia increased CERO2 and OCR in vivo and in vitro $(n=4)$. A There is an interaction effect between hypoxia treatment and hypercapnia treatment $(P<0.01)$. B Simple effects analyses show an increased CERO2 in Hypoxemia group ( ${ }^{*}<<0.05$ ), but not in Hypercapnia group ( $n s P>0.05$ ) compared with Sham group. HH group has the highest CERO2 levels as compared with Hypoxemia group $(\star \star \mathrm{P}<$ 0.01 ) and Hypercapnia group (** $\mathrm{P}<0.01$ ). $C$ The OCR levels are the highest in $12 \mathrm{~h}$ group as compared with other groups ( $12 \mathrm{~h}$ vs $0 \mathrm{~h}$ : $\mathrm{P}<0.01 ; 12 \mathrm{~h}$ vs $6 \mathrm{~h}$ : $\mathrm{P}>0.05 ; 12 \mathrm{~h}$ vs $24 \mathrm{~h}: \mathrm{P}<0.01)$. The $0 \mathrm{CR}$ levels are increased time-dependently and achieved a stationary phase at $18 \mathrm{~min}$. So the microglia cells were treated for 12 hours, and the OCR was examined at 18 min after the treatment, as shown in graph $D$ and E. D There is an interaction effect between $0.2 \% 02$ treatment and $15 \% \mathrm{CO} 2$ treatment $(P<0.01)$. E Simple effects analyses show increased levels of OCR in Hypoxia group ( $* \star P<0.01$ ), but not in HC group (ns $\mathrm{P}>0.05$ ) compared with Control group. Hypoxia $+\mathrm{HC}$ group has the highest levels of OCR as 
compared with Hypoxia group ( ${ }^{\star \star} P<0.01$ ) and HC group (** $P<0.01$ ). ns, non-significant; Sham group, sham-operated group; $\mathrm{HH}$ group, hypercapnia + hypoxemia group; $\mathrm{HC}$ group, high concentration of carbon dioxide group; $\mathrm{CERO} 2$, cerebral oxygen extraction rate; $\mathrm{OCR}$, oxygen consumption rate. The concentrations of 02 and $\mathrm{CO} 2$ in the air are $21 \%$ and $0.03 \%$.

A

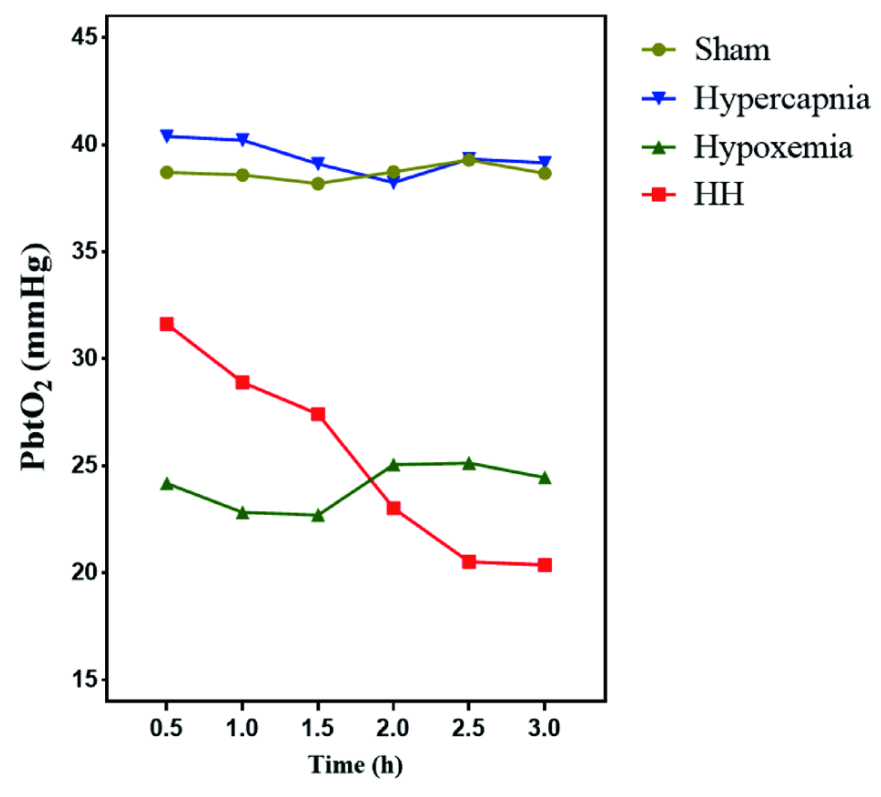

B

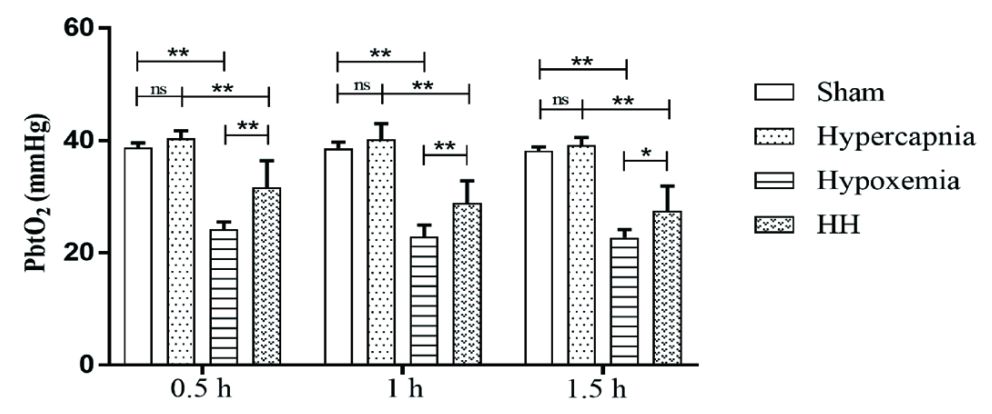

C

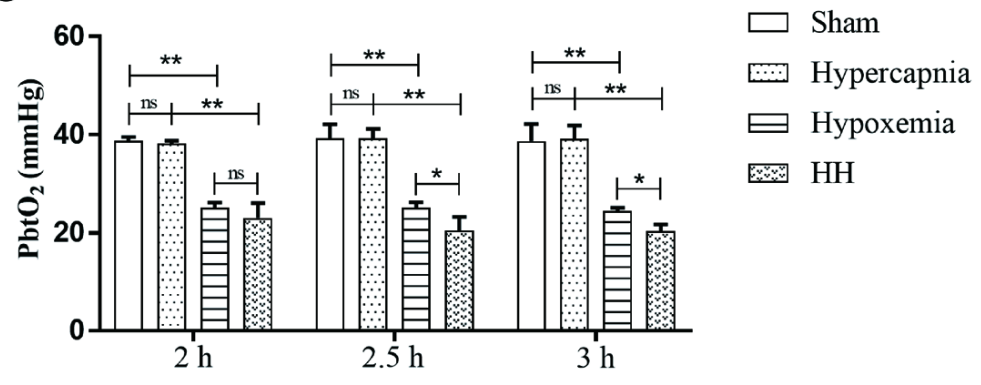

Figure 2

Hypercapnia decreased PbtO2 in hypoxemic rats $(n=4)$. Graphs A illustrate the PbtO2 at 0.5, 1, 1.5, 2, 2.5, and $3 \mathrm{~h}$ after ventilation in the rats. $\mathrm{B}, \mathrm{C}$ Compared with the Sham group, PbtO2 levels are decreased in Hypoxemia group $(0.5 \mathrm{~h}-3 \mathrm{~h}$ : $\mathrm{P}<0.01)$, but not in Hypercapnia group $(0.5 \mathrm{~h}-3 \mathrm{~h}: \mathrm{P}>0.05)$. HH group has higher PbtO2 levels as compared with Hypoxemia group in the first 1.5 hours $(0.5 \mathrm{~h}: \mathrm{P}<0.01 ; 1 \mathrm{~h}: \mathrm{P}<$ $0.01 ; 1.5 \mathrm{~h}$ : $\mathrm{P}<0.05)$. On the contrary, $\mathrm{HH}$ group has lower PbtO2 levels as compared with Hypoxemia group after 2 hours (2.5 h: $\mathrm{P}<0.05 ; 3$ h: $\mathrm{P}<0.05)$. Sham group, sham-operated group; HH group, hypercapnia + hypoxemia group; Pbt02, partial pressure of brain tissue oxygen. 

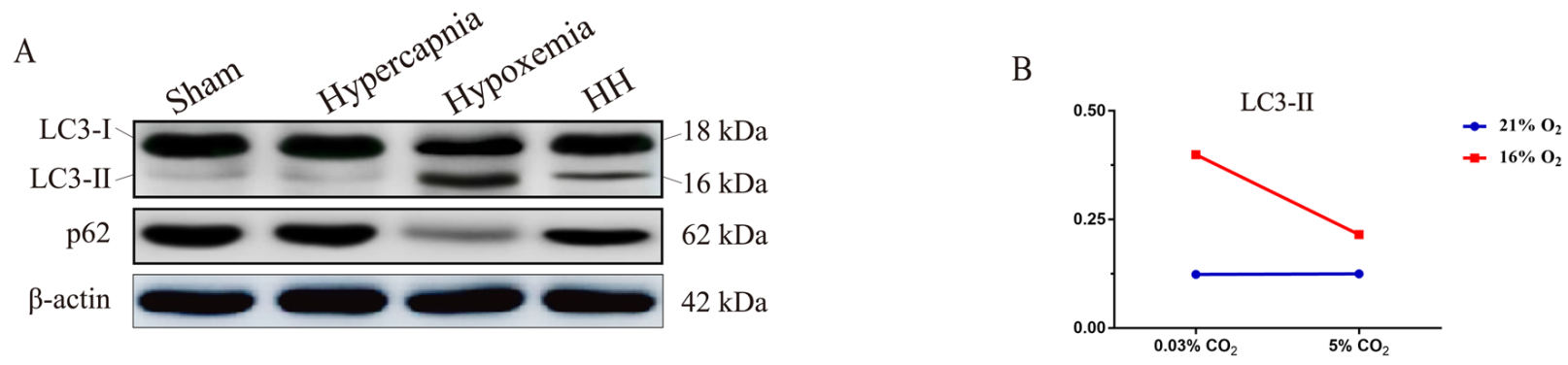

$\mathrm{C}$

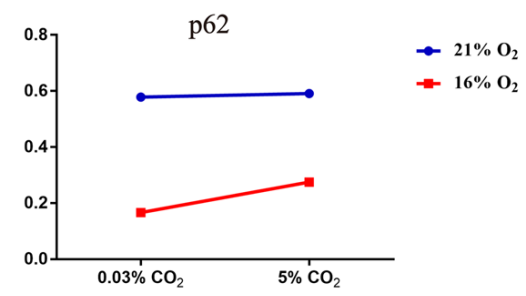

$\mathrm{F}$
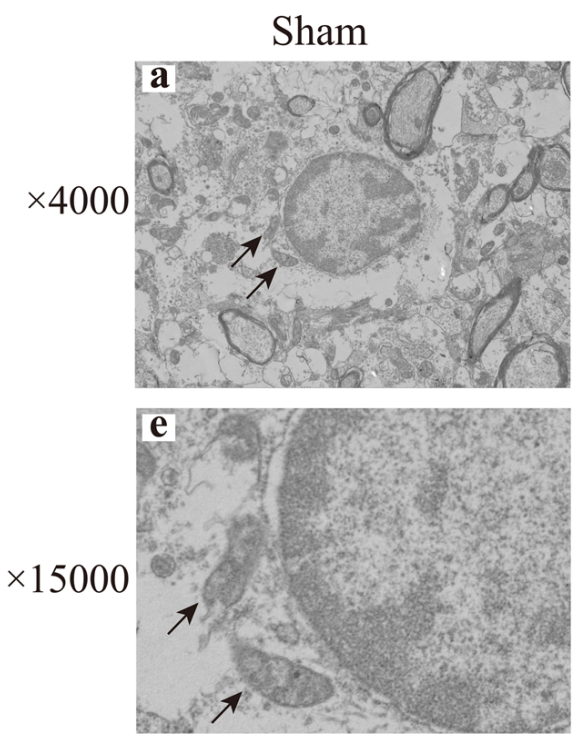

$\mathrm{D}$

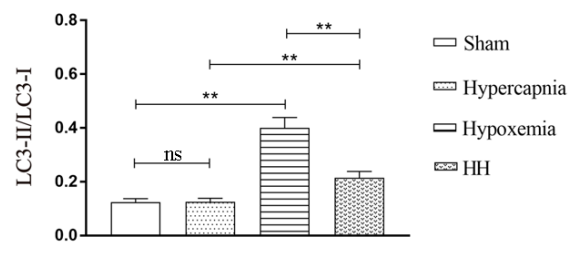

$\mathrm{E}$

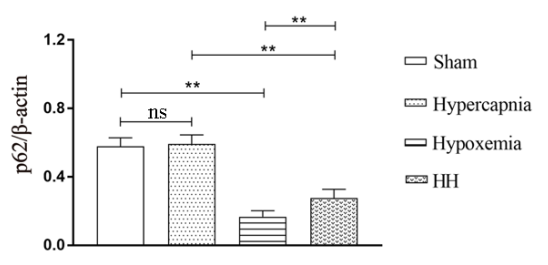

Hypercapnia
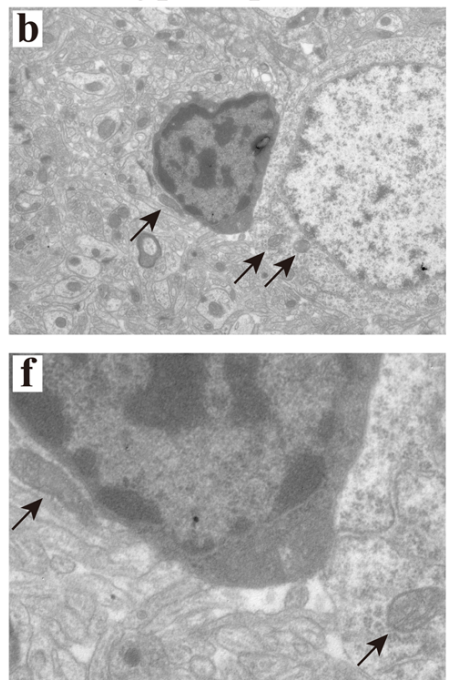

Hypoxemia
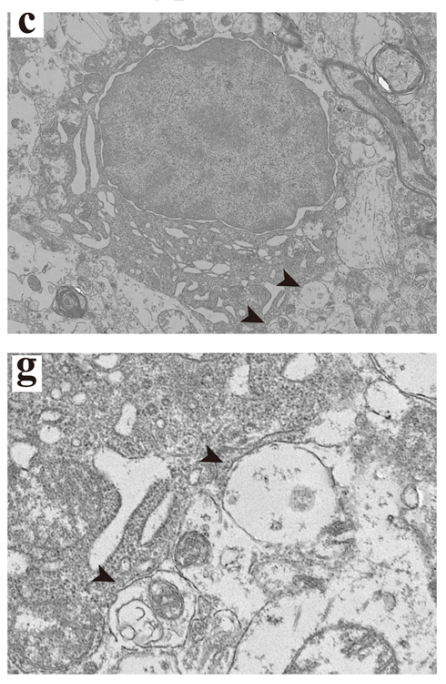

$\mathrm{HH}$
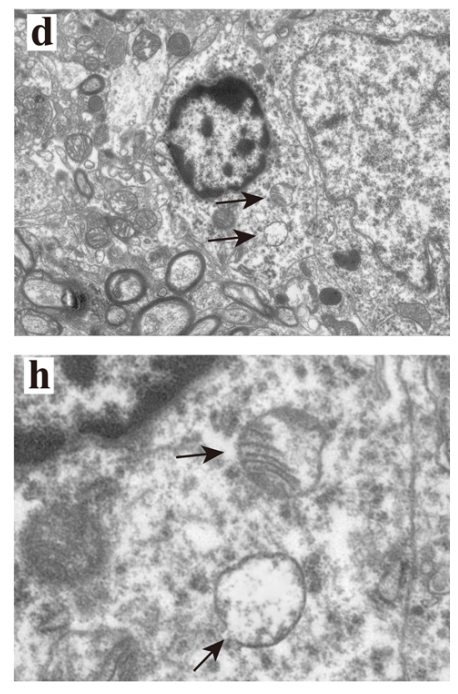

\section{Figure 3}

Hypercapnia inhibited mitophagy in microglia in the hypoxic hippocampus $(n=4)$. A Immunoreactive bands of LC3-I (18 kDa), LC3-II (16 kDa), p62 (62 kDa), and $\beta$-actin (42 kDa). B, C There is an interaction effect between hypoxia treatment and hypercapnia treatment (LC3-II: $P<0.01$, p62: $P<0.01)$. D Simple effects analyses show increased protein expression levels of LC3-II in Hypoxemia group compared with Sham group (** $\mathrm{P}<0.01$ ). HH group shows lower expression levels of LC3-II in comparison with Hypoxemia group $(\star \star P<0.01)$. E In contrast, a significant decrease in p62 expression is observed in Hypoxemia group compared with Sham group $(\star \star \mathrm{P}<0.01)$. The levels of $\mathrm{p} 62$ expression in $\mathrm{HH}$ group are higher compared with Hypoxemia group ( $* * P<0.01)$. F Transmission electron microscope images showing ultrastructural alterations of microglia in the hypoxic hippocampus with hypercapnia treatment. The mitochondria exhibit normal morphology (arrow) in sham group (a, e) and hypercapnia group (b, $f$ ). The hypoxic microglia show the formation of abundant autophagic vacuoles enveloping mitochondria 
(arrowhead) $(c, g)$. There are swollen mitochondria in hypercapnia + hypoxia treated microglia (arrow). However, autophagic vacuoles are rarely observed $(d, h)$. ns, non-significant; Sham group, sham-operated group; $\mathrm{HH}$ group, hypercapnia + hypoxemia group. The concentrations of 02 and $\mathrm{CO} 2$ in the air are $21 \%$ and $0.03 \%$.
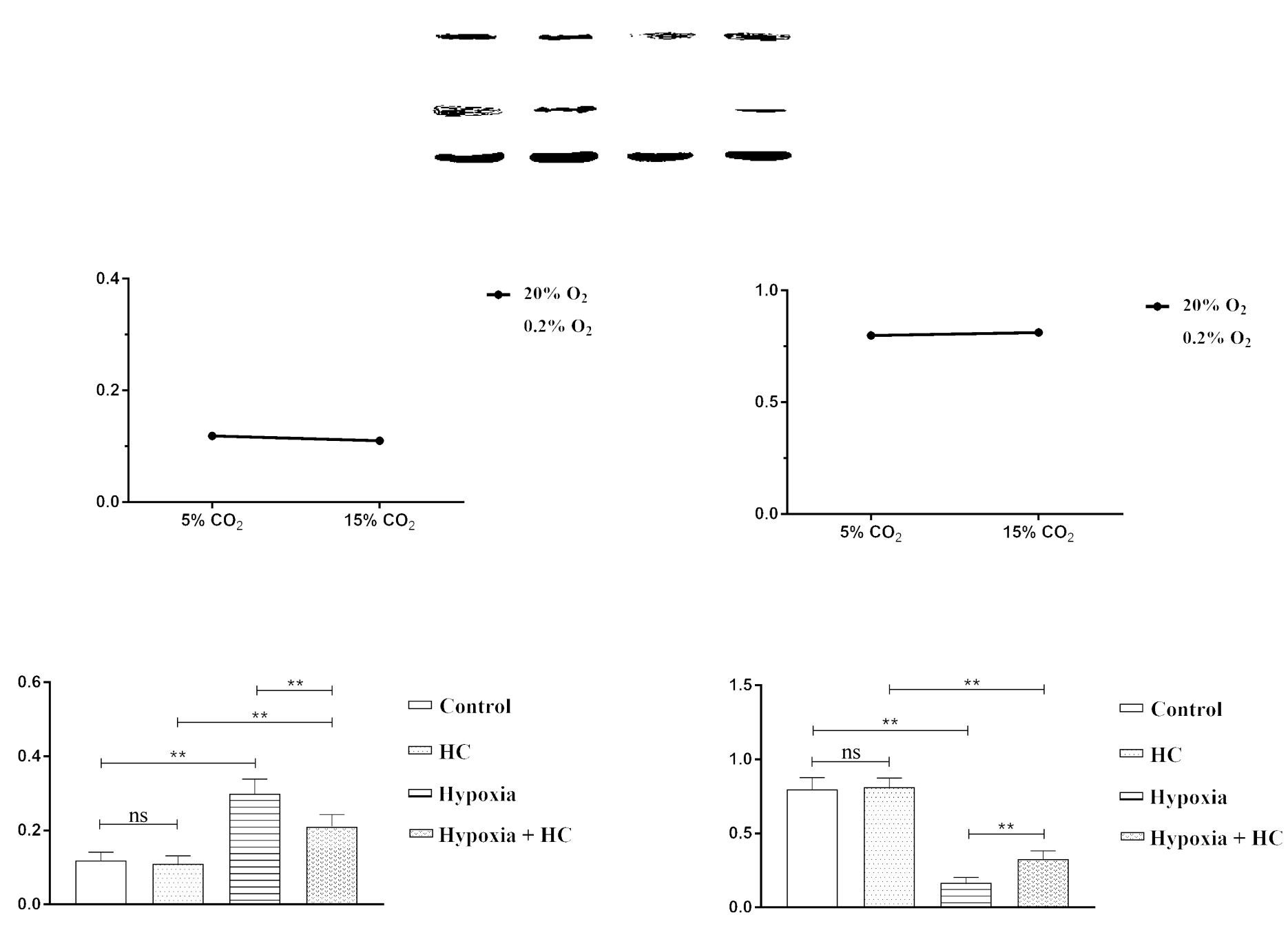

\section{Figure 4}

$15 \%$ CO2 inhibited mitophagy in hypoxia-activated BV-2 microglia $(n=4)$. A Immunoreactive bands of LC3-I (18 kDa), LC3-II (16 kDa), p62 (62 kDa), and $\beta$-actin (42 kDa). B, C There is an interaction effect between $0.2 \% 02$ treatment and 15\% CO2 treatment (LC3-II: $P<0.01$, p62: $P<0.01$ ) . D Simple effects analyses show increased protein expression levels of LC3-II in Hypoxia group compared with Control group $(P<0.01)$. Hypoxia $+\mathrm{HC}$ group shows lower expression levels of LC3-II in comparison with Hypoxia group $(P<0.01)$. E In contrast, a significant decrease in p62 expression is observed in Hypoxia group compared with Control group $(P<0.01)$. The levels of p62 expression in Hypoxia $+\mathrm{HC}$ group are higher compared with Hypoxia group $(\mathrm{P}<0.01)$. ns, non-significant; $\mathrm{HC}$ group, high concentration of carbon dioxide group. 
A

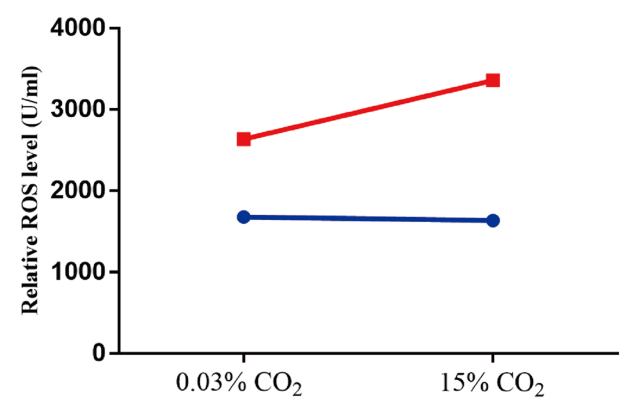

B

$-21 \% \mathrm{O}_{2}$
$-\quad 16 \% \mathrm{O}_{2}$

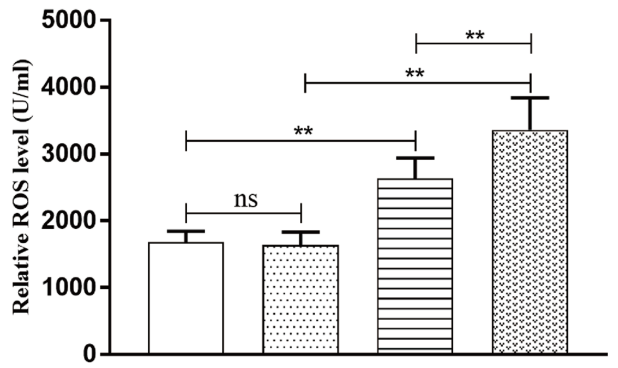

$\square$ Sham

[-i. Hypercapnia

Е Hypoxemia

E:्च $\mathrm{HH}$

C
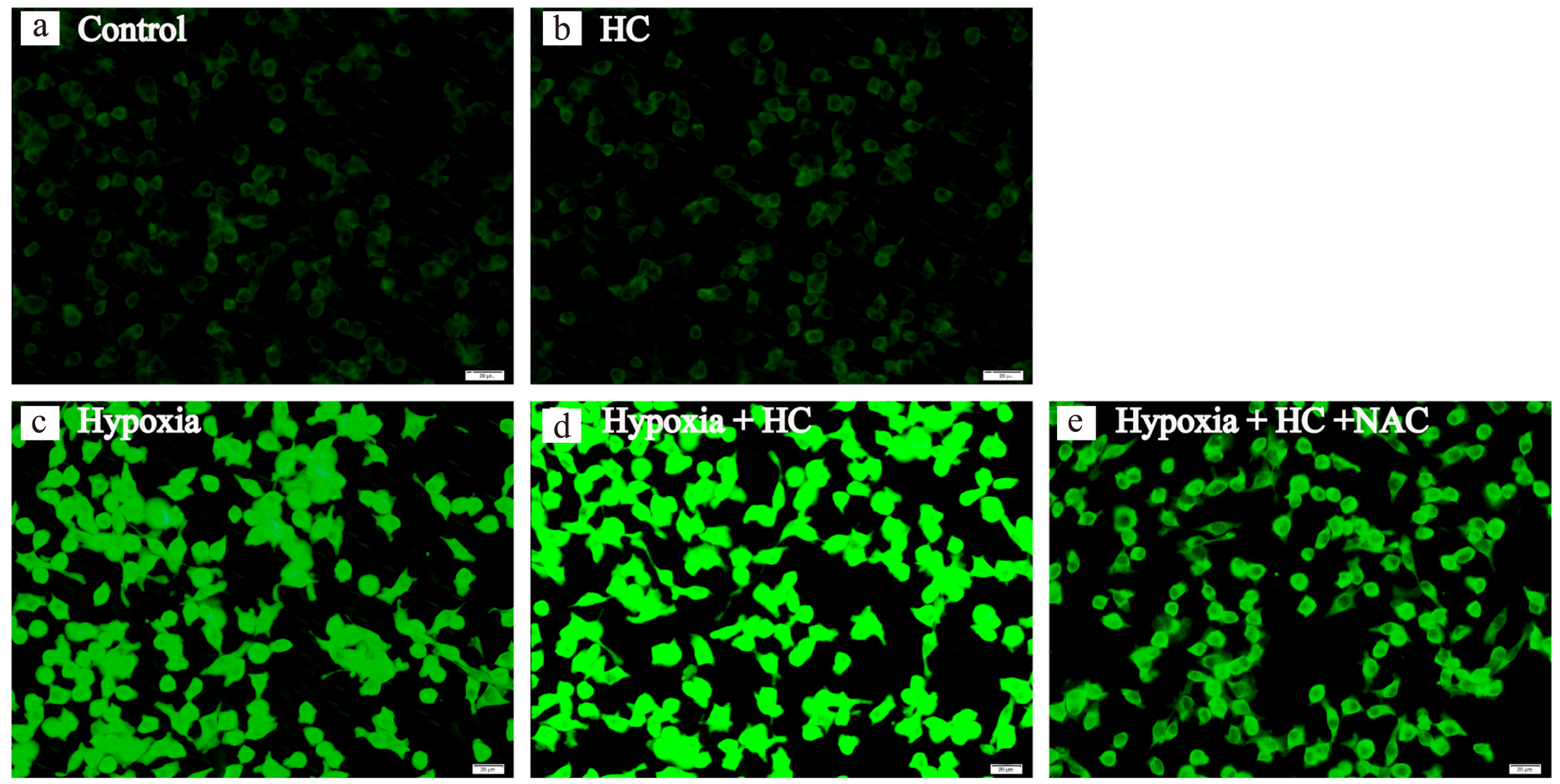

Figure 5

Hypercapnia induced overproduction of ROS in the hypoxemic hippocampus and BV2 cells $(n=4)$. A There is an interaction effect between hypoxia treatment and hypercapnia treatment $(P<0.05)$. B Simple effects analyses show increased levels of ROS in Hypoxemia group ( $* \star P<0.01$ ), but not in Hypercapnia group ( $\mathrm{ns} \mathrm{P}>0.05$ ) compared with Sham group. HH group has the highest ROS levels as compared with Hypoxemia group $(* \star P<0.01)$ and Hypercapnia group $(* \star P<0.01)$. C Immunofluorescence images showing the production of ROS (a-e, green). Enhanced ROS immunofluorescence is evident in Hypoxia group, but not in $\mathrm{HC}$ group compared with Control group. Hypoxia + HC group exhibits the most intense ROS fluorescence as compared with Hypoxia group and HC group. ROS fluorescence is noticeably reduced with NAC pretreatment (2 mM) in BV-2 microglial cells. Scale bars: 20 um. ns, non-significant; Sham group, sham-operated group; HH group, hypercapnia + hypoxemia group; ROS, reactive oxygen 
species; $\mathrm{HC}$ group, high concentration of carbon dioxide group. The concentrations of 02 and $\mathrm{CO} 2$ in the air are $21 \%$ and $0.03 \%$.
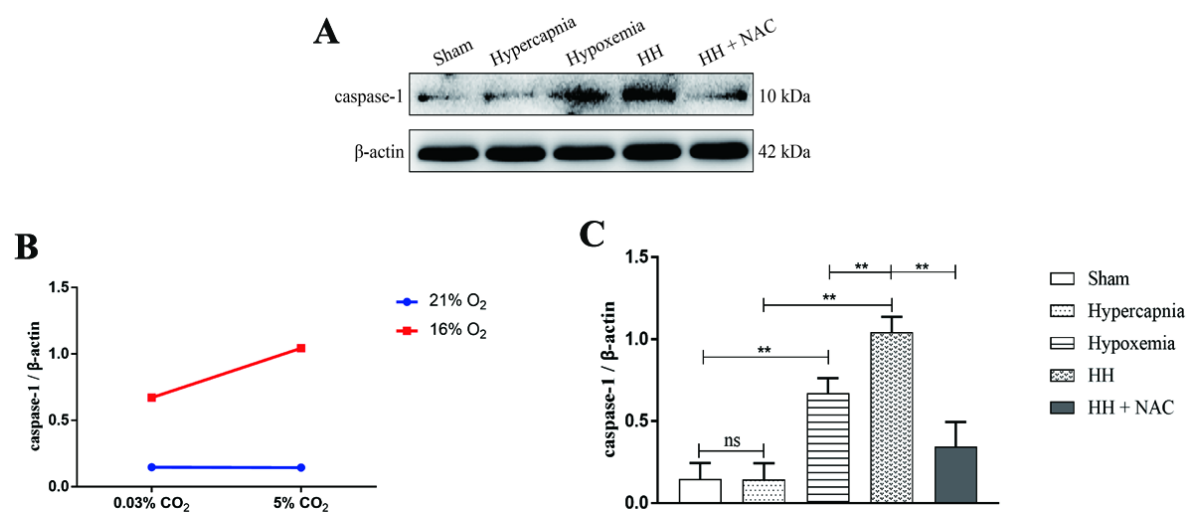

D
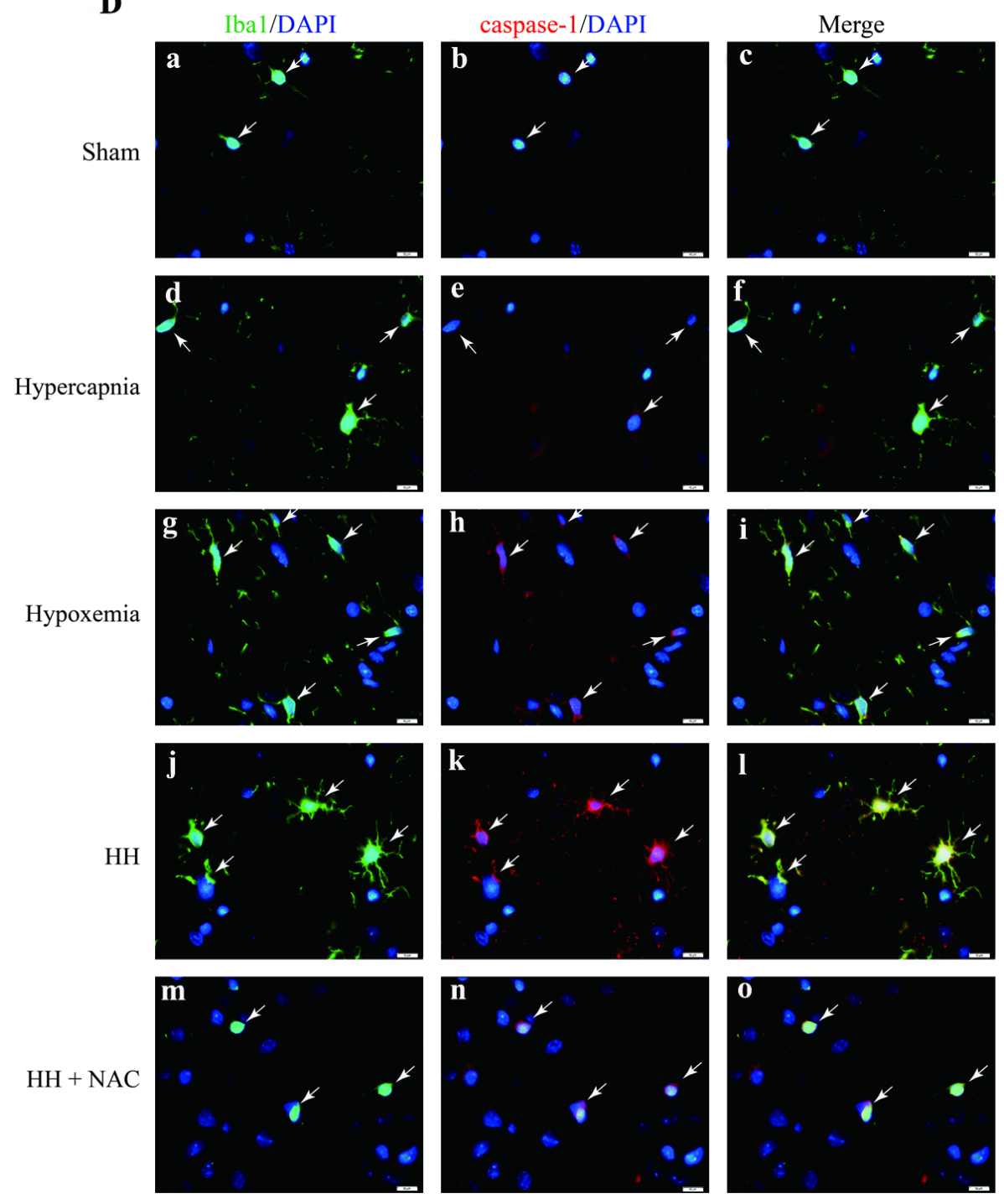

Figure 6

Hypercapnia enhanced NLRP3 inflammasome activation via inducing ROS overproduction in microglia of the hypoxic hippocampus $(n=4)$. A Immunoreactive bands of caspase-1 (10 kDa) and $\beta$-actin $(42 \mathrm{kDa})$. B There is an interaction effect between hypoxia treatment and hypercapnia treatment $(P<0.01)$. C Simple 
effects analyses show increased protein expression levels of caspase-1 in Hypoxemia group (** $P<0.01$ ), but not in Hypercapnia group ( $\mathrm{ns} \mathrm{P}>0.05$ ) compared with Sham group. $\mathrm{HH}$ group has the highest expression levels of caspase-1 as compared with Hypoxemia group ( $* * P<0.01)$ and Hypercapnia group ( $\star * \mathrm{P}<0.01)$. The protein expression of caspase-1 is significantly suppressed with NAC pretreatment (150 $\mathrm{mg} / \mathrm{kg})$ in rats $(* * P<0.01)$. D Immunofluorescence images showing the expression of Iba1+ microglia $(a$, $d, g, j, m$, green), caspase- 1 (b, e, h, k, n, red), and the co-localization of caspase- 1 and microglia ( $x, f, i, l$, o). Enhanced caspase-1 immunofluorescence is evident in Hypoxemia group, but not in Hypercapnia group compared with Sham group. HH group emits the strongest caspase-1 fluorescence as compared with Hypoxemia group and Hypercapnia group. Caspase-1 fluorescence is markedly attenuated in rats given NAC pretreatment. Scale bars: $10 \mu \mathrm{m}$. ns, non-significant; Sham group, sham-operated group; $\mathrm{HH}$ group, hypercapnia + hypoxemia group. The concentrations of 02 and $\mathrm{CO} 2$ in the air are $21 \%$ and $0.03 \%$. 

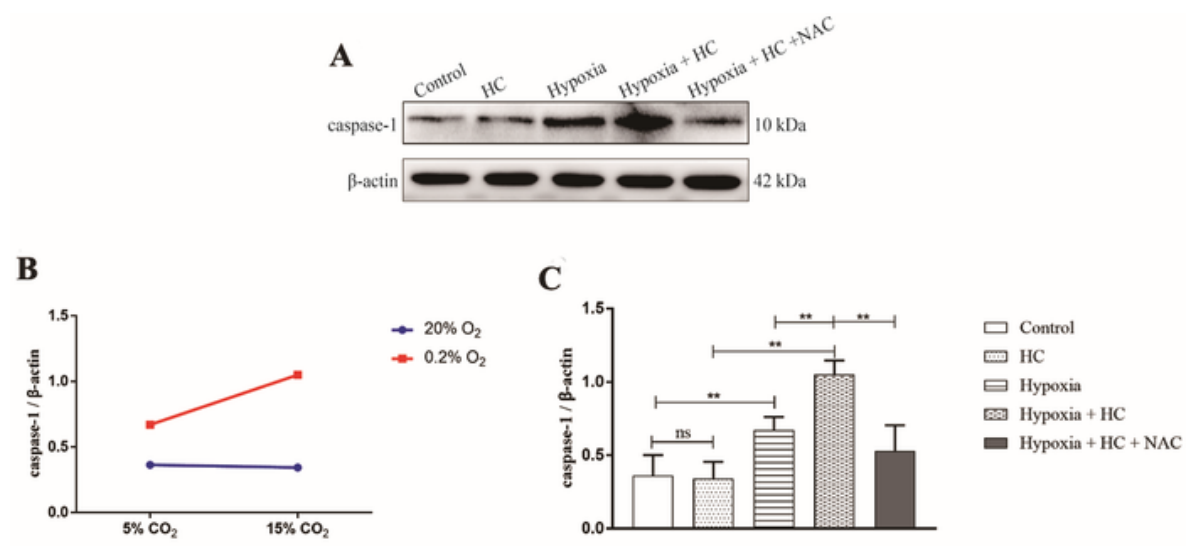

D
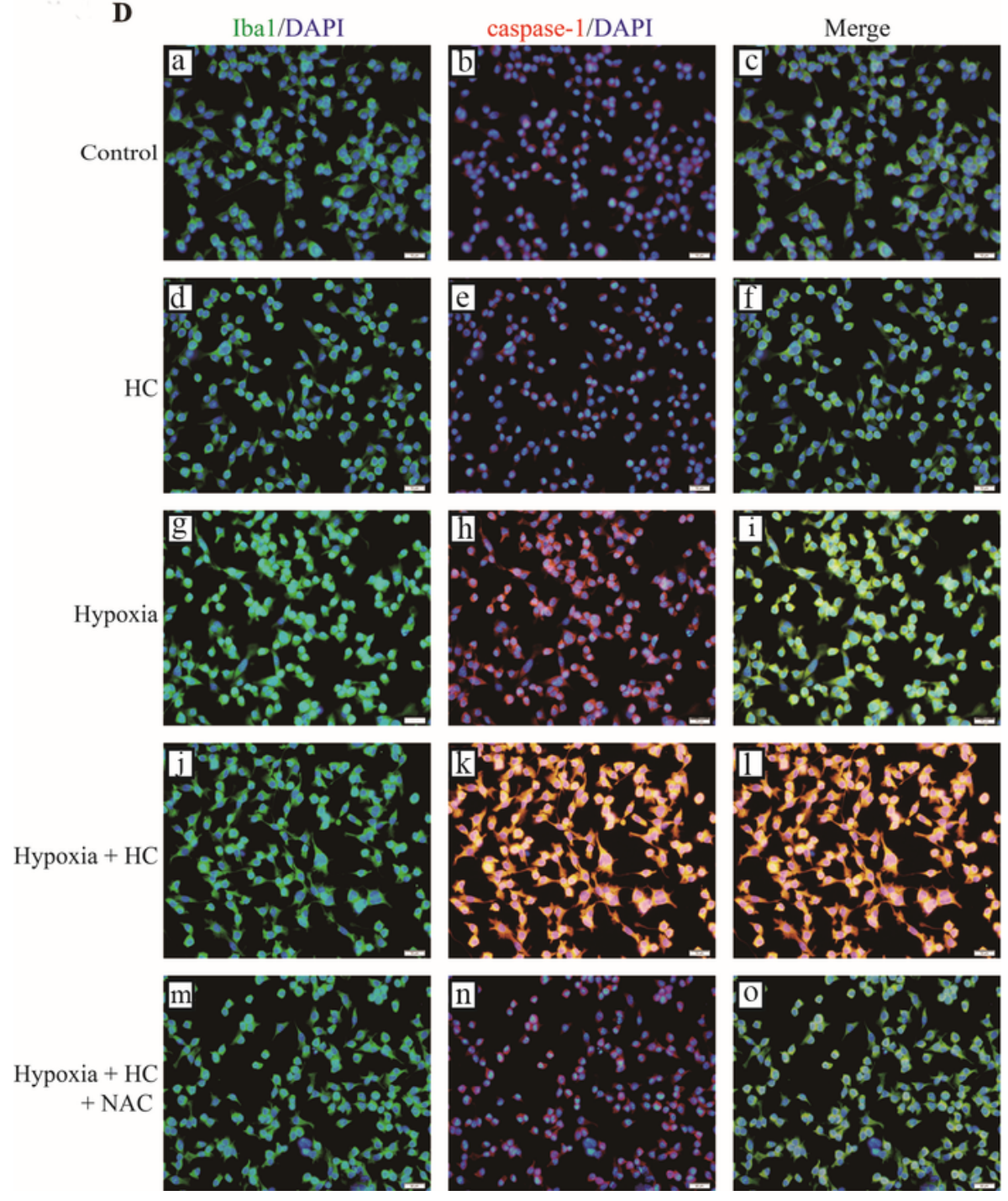

Figure 7

$15 \%$ CO2 enhanced NLRP3 inflammasome activation via inducing ROS overproduction in hypoxic BV-2 cells $(n=4)$. A Immunoreactive bands of caspase- $1(10 \mathrm{kDa})$ and $\beta$-actin $(42 \mathrm{kDa})$. B There is an interaction effect between $0.2 \% 02$ treatment and $15 \%$ CO2 treatment $(P<0.01)$. C Simple effects analyses show increased protein expression levels of caspase- 1 in Hypoxia group ( $* \star P<0.01)$, but not in $\mathrm{HC}$ group (ns $\mathrm{P}>0.05$ ) compared with Control group. Hypoxia + HC group shows the highest expression 
levels of caspase-1 in comparison with Hypoxia group (** $\mathrm{P}<0.01$ ) and $\mathrm{HC}$ group (** $\mathrm{P}<0.01$ ). Additionally, the protein expression of caspase-1 is significantly suppressed with NAC pretreatment (2 $\mathrm{mM}$ ) in BV-2 microglial cells (** $\mathrm{P}<0.01)$. D Immunofluorescence images showing the expression of lba1+ microglia (a, d, g, j, m, green), caspase-1 (b, e, h, k, n, red), and the co-localization of caspase-1 and microglia ( $\mathrm{x}, \mathrm{f}, \mathrm{i}, \mathrm{l}, \mathrm{o})$. Enhanced caspase-1 immunofluorescence is evident in Hypoxia group, but not in $\mathrm{HC}$ group compared with Control group. Hypoxia $+\mathrm{HC}$ group shows the strongest caspase-1 fluorescence as compared with Hypoxia group and $\mathrm{HC}$ group. Caspase-1 fluorescence is drastically reduced with NAC pretreatment (2 mM) in BV-2 microglial cells. Scale bars: $10 \mu \mathrm{m}$. ns, non-significant; HC group, high concentration of carbon dioxide group.

A

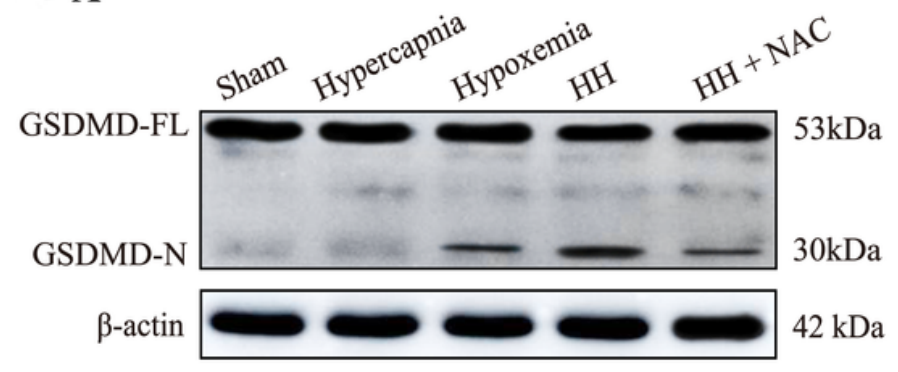

B

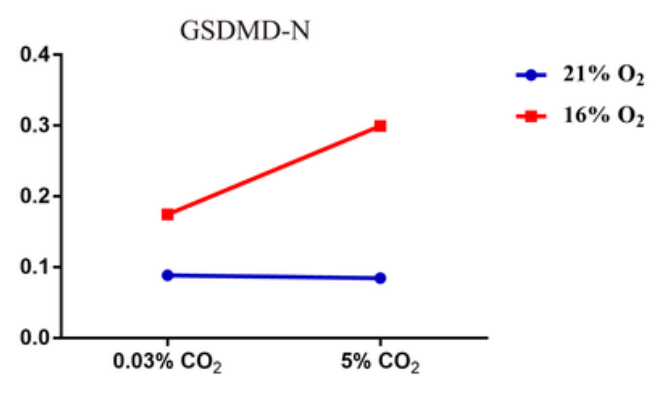

C

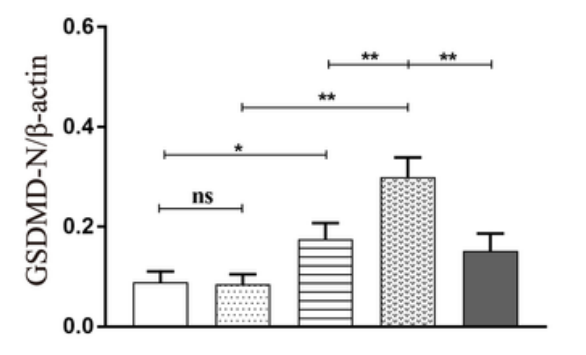

D

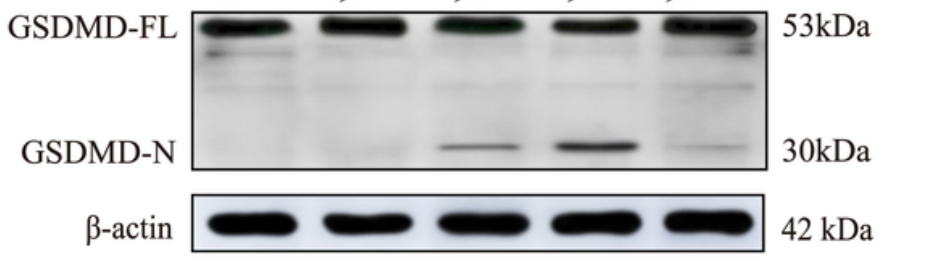

$\mathbf{E}$

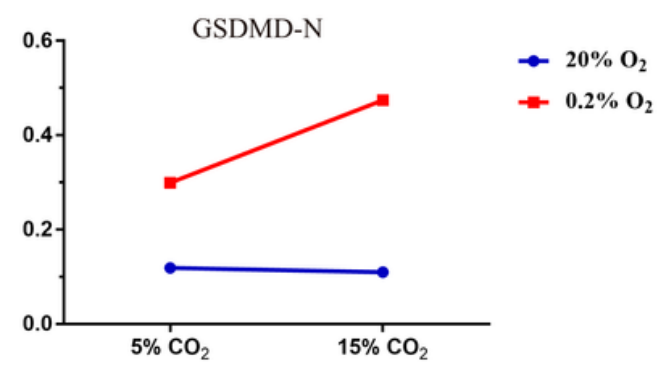

$\mathbf{F}$

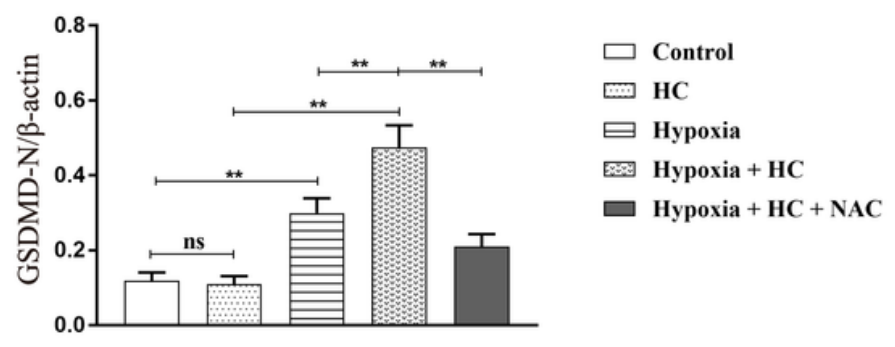

\section{Figure 8}

Hypercapnia increased GSDMD-N expression via inducing ROS overproduction in microglia in vivo and in vitro. A, D Immunoreactive bands of GSDMD-FL (53 kDa), GSDMD-N (30 kDa) and $\beta$-actin (42 kDa). B There is an interaction effect between hypercapnia treatment and hypoxia treatment $(P<0.01)$. E There is 
an interaction effect between $0.2 \% 02$ treatment and $15 \%$ CO2 treatment ( $* * P<0.01$ ). C Simple effects analyses show increased protein expression levels of GSDMD-N in Hypoxemia group ( ${ }^{*}<<0.05$ ), but not in Hypercapnia group ( $\mathrm{ns} \mathrm{P}>0.05$ ) compared with Sham group. HH group shows the highest expression levels of GSDMD-N in comparison with Hypoxemia group ( $\left.{ }^{\star *} \mathrm{P}<0.01\right)$ and Hypercapnia group $(* \star \mathrm{P}<$ $0.01)$. Additionally, the protein expression of GSDMD-N is significantly suppressed with NAC pretreatment in rats $(* * P<0.01)$. F Simple effects analyses show increased protein expression levels of GSDMD-N in Hypoxia group ( $\star \star \mathrm{P}<0.01$ ), but not in $\mathrm{HC}$ group (ns $\mathrm{P}>0.05$ ) compared with Control group. Hypoxia + $\mathrm{HC}$ group has the highest expression levels of GSDMD-N as compared with Hypoxia group $(* \star P<0.01)$ and $\mathrm{HC}$ group (** $\mathrm{P}<0.01$ ). Additionally, the protein expression of GSDMD-N is significantly suppressed with NAC pretreatment in BV-2 microglial cells ( $* * P<0.01$ ). DSDMD-FL, gasdermin D-full length; GSDMD$\mathrm{N}$, gasdermin D-N domains. ns, non-significant; Sham group, sham-operated group; HH group, hypercapnia + hypoxemia group; $\mathrm{HC}$ group, high concentration of carbon dioxide group. The concentrations of 02 and $\mathrm{CO} 2$ in the air are $21 \%$ and $0.03 \%$. 
A

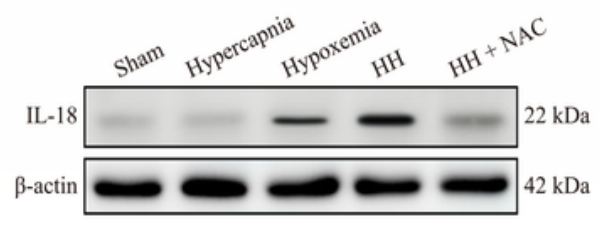

B

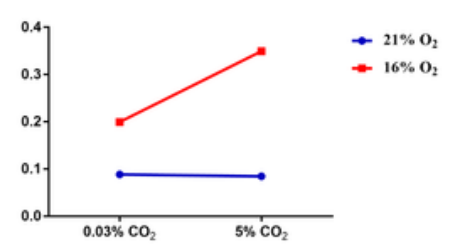

$\mathrm{D}$
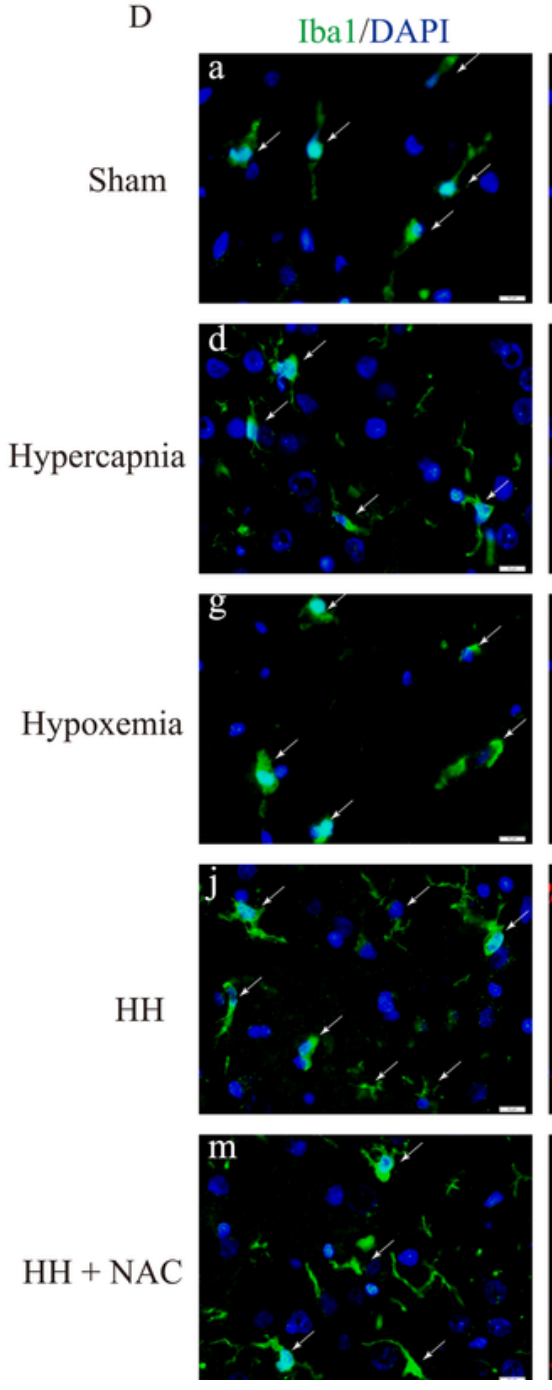

C

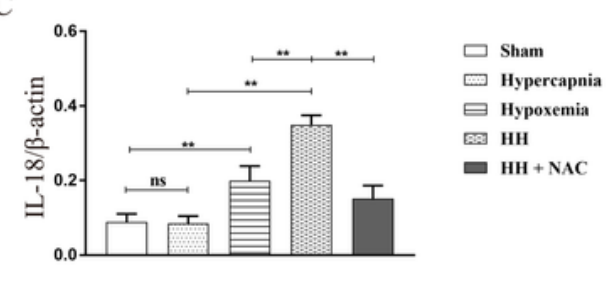

IL-18/DAPI
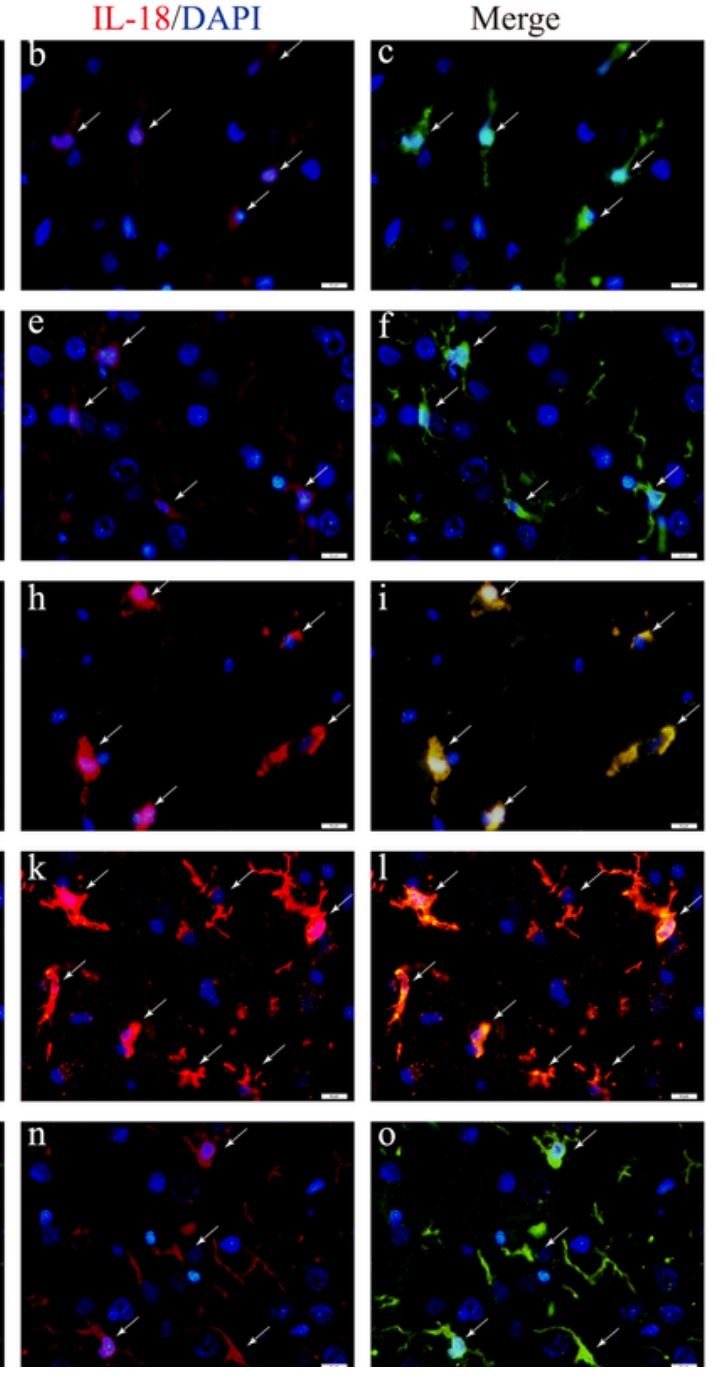

\section{Figure 9}

Hypercapnia increased IL-1 $\beta$ expression via inducing ROS overproduction in microglia of hypoxic hippocampus $(n=4)$. A Immunoreactive bands of IL-1 $\beta(17 \mathrm{kDa})$ and $\beta$-actin $(42 \mathrm{kDa})$. B There is an interaction effect between hypoxia treatment and hypercapnia treatment $(P<0.01)$. C Simple effects analyses show increased protein expression levels of IL-1 $\beta$ in Hypoxemia group ( $* \star P<0.01$ ), but not in Hypercapnia group (ns $\mathrm{P}>0.05$ ) compared with Sham group. HH group has the highest expression levels 
of IL-1 $\beta$ as compared with Hypoxemia group (** $P<0.01$ ) and Hypercapnia group (** $P<0.01)$. The protein expression of IL-1 $\beta$ is significantly suppressed with NAC pretreatment in rats $(* * P<0.01)$. D Immunofluorescence images showing the expression of Iba $1+$ microglia $(a, d, g, j, m$, green), IL-1 $\beta$ (b, e, h, $k, n$, red), and the co-localization of IL-1 $\beta$ and microglia ( $x, f, i, I, 0)$. Enhanced IL-1 $\beta$ immunofluorescence is evident in Hypoxemia group, but not in Hypercapnia group compared with Sham group. HH group has the strongest IL-1 $\beta$ fluorescence as compared with Hypoxemia group and Hypercapnia group. IL-1 $\beta$ fluorescence is markedly reduced in rats given NAC pretreatment. Scale bars: $10 \mu \mathrm{m}$. IL-1 1 , interleukin-1 beta; ns, non-significant; Sham group, sham-operated group; HH group, hypercapnia + hypoxemia group. The concentrations of $\mathrm{O} 2$ and $\mathrm{CO} 2$ in the air are $21 \%$ and $0.03 \%$. 

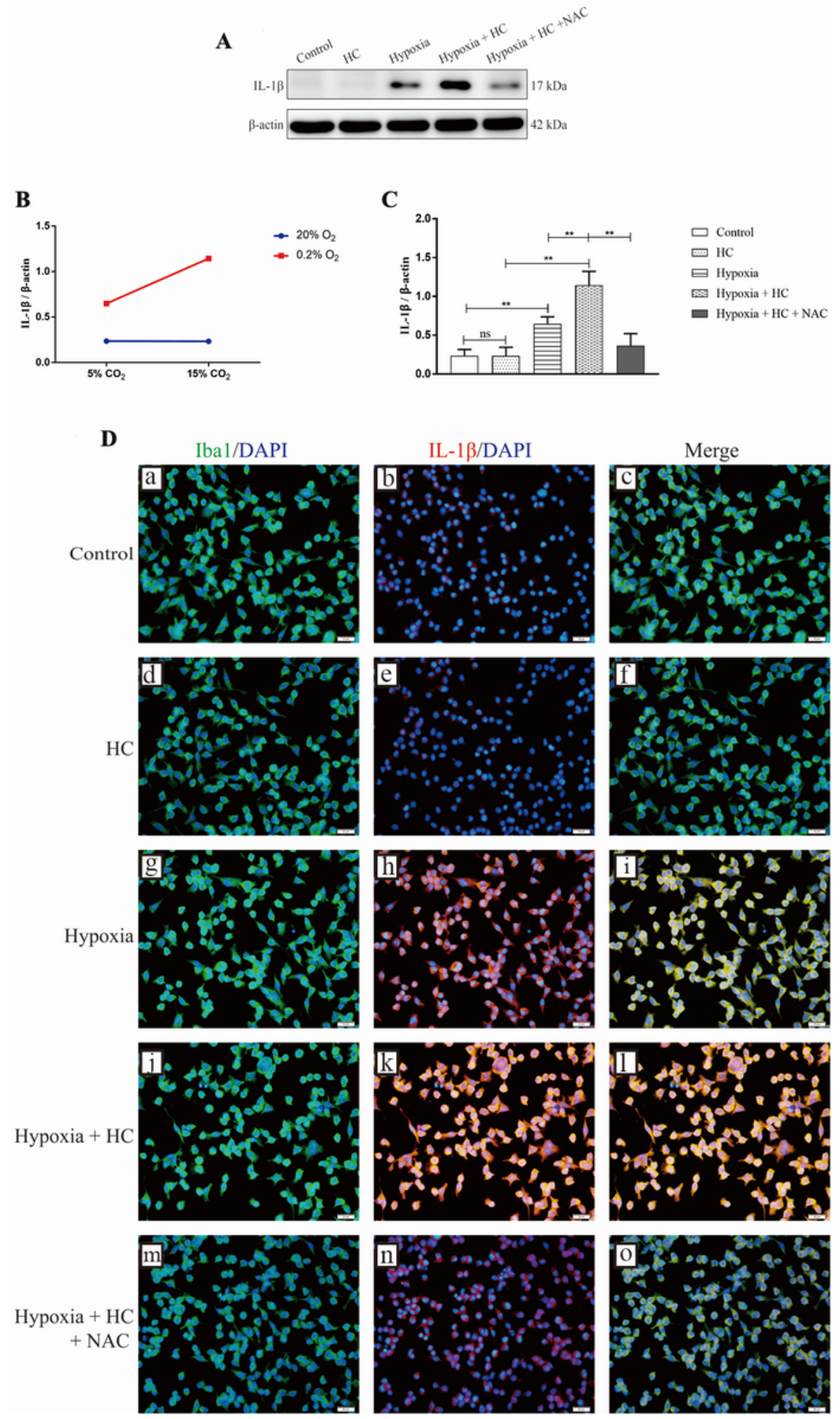

Figure 10

$15 \%$ CO2 increased IL-1 $\beta$ expression via inducing ROS overproduction in hypoxic BV-2 cells $(n=4)$. A Immunoreactive bands of IL-1 $\beta(17 \mathrm{kDa})$ and $\beta$-actin (42 kDa). B There is an interaction effect between $0.2 \% 02$ treatment and $15 \% \mathrm{CO} 2$ treatment $(P<0.01)$. C Simple effects analyses show increased protein expression levels of IL-1 $\beta$ in Hypoxia group (** $\mathrm{P}<0.01$ ), but not in $\mathrm{HC}$ group ( $\mathrm{ns} \mathrm{P}>0.05$ ) compared with Control group. Hypoxia + HC group shows the highest expression levels of IL-1 $\beta$ as compared with 
Hypoxia group (** $\mathrm{P}<0.01$ ) and $\mathrm{HC}$ group (** $\mathrm{P}<0.01$ ). Additionally, the protein expression of IL-1 $\beta$ is significantly suppressed with NAC pretreatment in BV-2 microglial cells ( $* * \quad P<0.01$ ). D

Immunofluorescence images showing Iba1+ microglia (a, d, g, j, m, green), IL-1 $\beta$ labeling (b, e, h, k, n, red), and their co-localization $(x, f, i, I, 0)$. Enhanced IL-1 $\beta$ immunofluorescence is evident in Hypoxia group, but not in $\mathrm{HC}$ group compared with Control group. Hypoxia $+\mathrm{HC}$ group displays the strongest IL-1 $\beta$ fluorescence when compared with Hypoxia group and HC group. IL-1 $\beta$ fluorescence is markedly reduced with NAC pretreatment ( $2 \mathrm{mM}$ ) in BV-2 microglial cells. Scale bars: $10 \mu \mathrm{m}$. IL-1 $\beta$, interleukin-1 beta; ns, non-significant; $\mathrm{HC}$ group, high concentration of carbon dioxide group. 
A

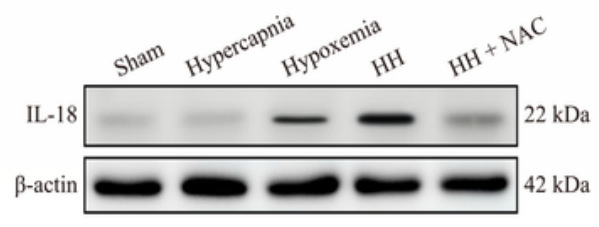

B

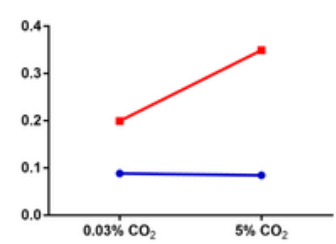

D

C

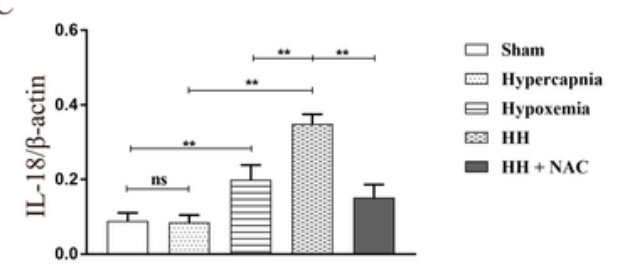

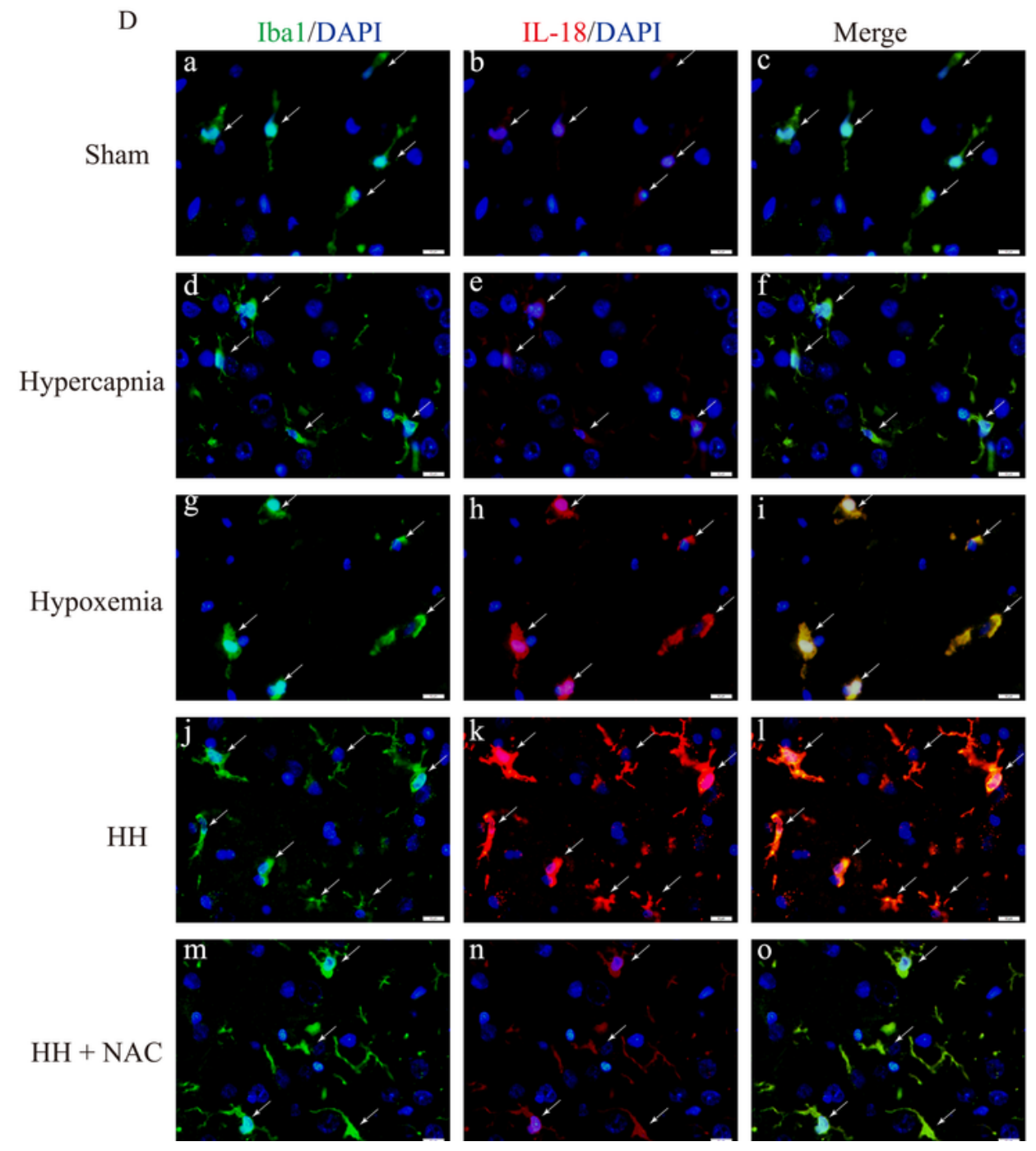
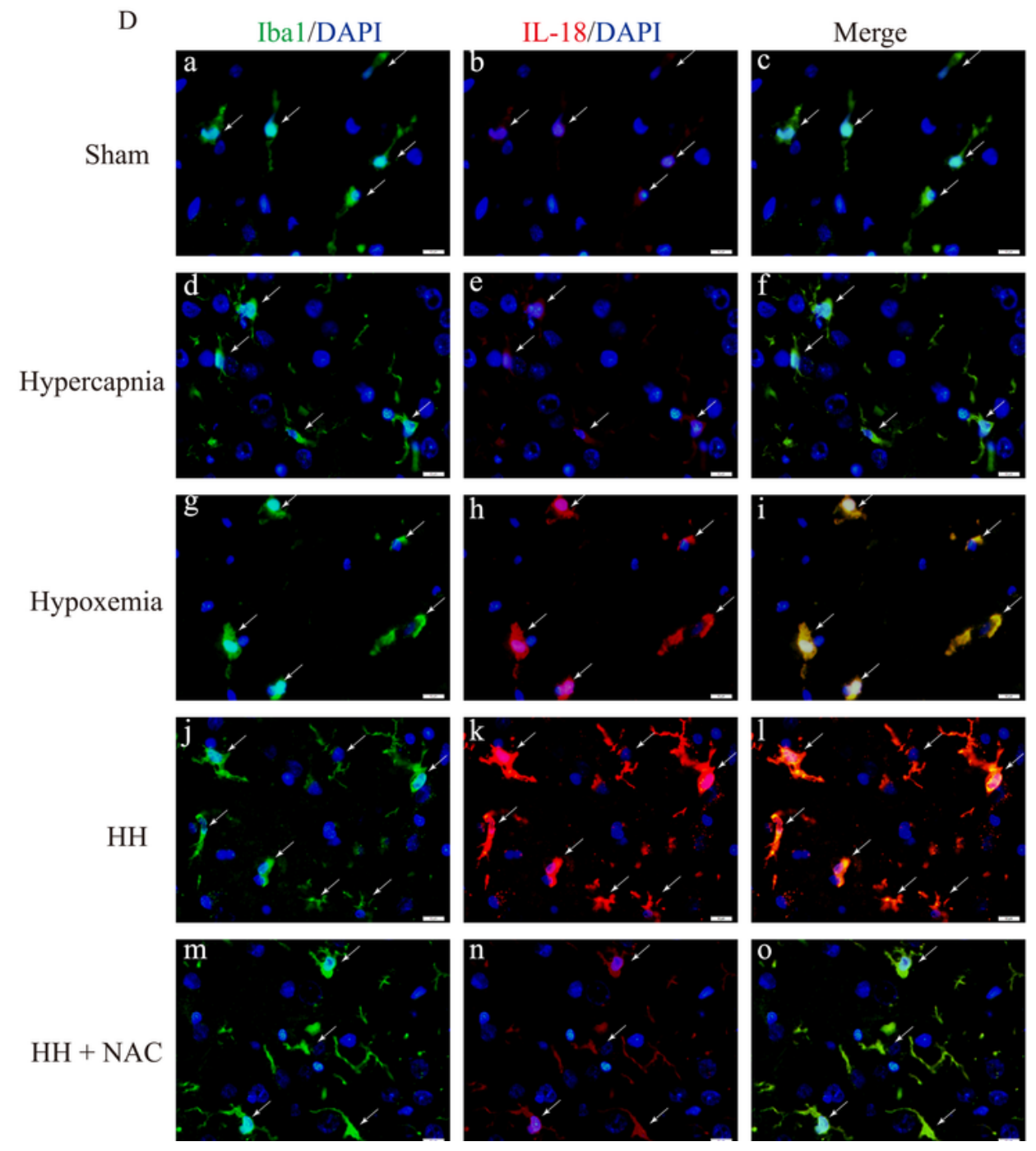

\section{Figure 11}

Hypercapnia increased IL-18 expression via inducing ROS overproduction in microglia of hypoxic hippocampus $(n=4)$. A Immunoreactive bands of IL-18 $(22 \mathrm{kDa})$ and $\beta$-actin $(42 \mathrm{kDa})$. B There is an interaction effect between hypoxia treatment and hypercapnia treatment $(P<0.01)$. C Simple effects analyses show increased protein expression levels of IL-18 in Hypoxemia group (** $P<0.01$ ), but not in Hypercapnia group (ns P > 0.05) compared with Sham group. HH group has the highest expression levels 
of IL-18 as compared with Hypoxemia group (** $P<0.01$ ) and Hypercapnia group (** $P<0.01)$. The protein expression of IL-18 is significantly suppressed with NAC pretreatment in rats $(* * P<0.01)$. D Immunofluorescence images showing the expression of Iba1+ microglia ( $a, d, g, j, m$, green), IL-18 (b, e, h, $k, n$, red), and the co-localization of IL-18 and microglia ( $x, f, i, I, o)$. Enhanced IL-18 immunofluorescence is evident in Hypoxemia group, but not in Hypercapnia group compared with Sham group. HH group has the strongest IL-18 fluorescence as compared with Hypoxemia group and Hypercapnia group. IL-18 fluorescence is markedly reduced in rats given NAC pretreatment. Scale bars: $10 \mu \mathrm{m}$. IL-18, interleukin-18; ns, non-significant; Sham group, sham-operated group; HH group, hypercapnia + hypoxemia group. The concentrations of 02 and $\mathrm{CO} 2$ in the air are $21 \%$ and $0.03 \%$. 
A

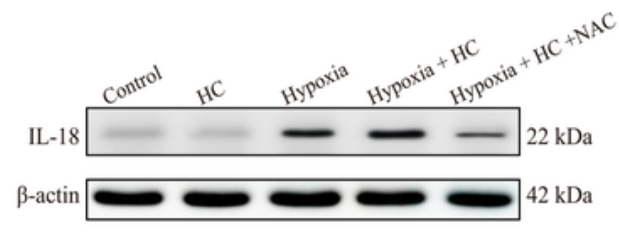

B

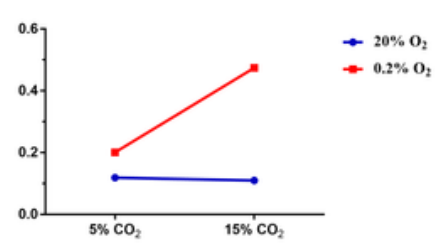

D
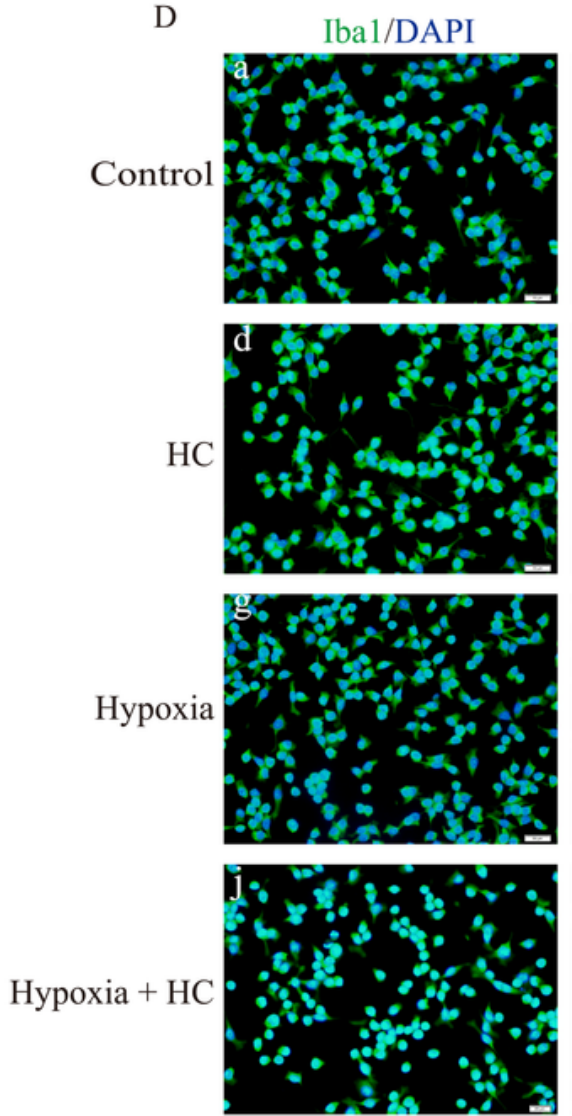

Hypoxia $+\mathrm{HC}$

$+\mathrm{NAC}$

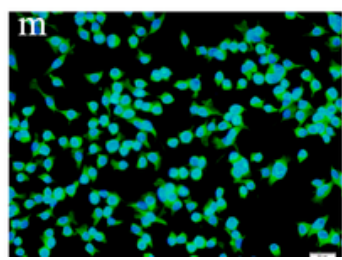

C

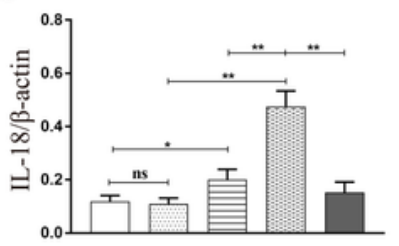

$\square$ Control

$\square$ HC

Hypoxia + HC

- Hypoxia $+\mathrm{HC}+\mathrm{NAC}$
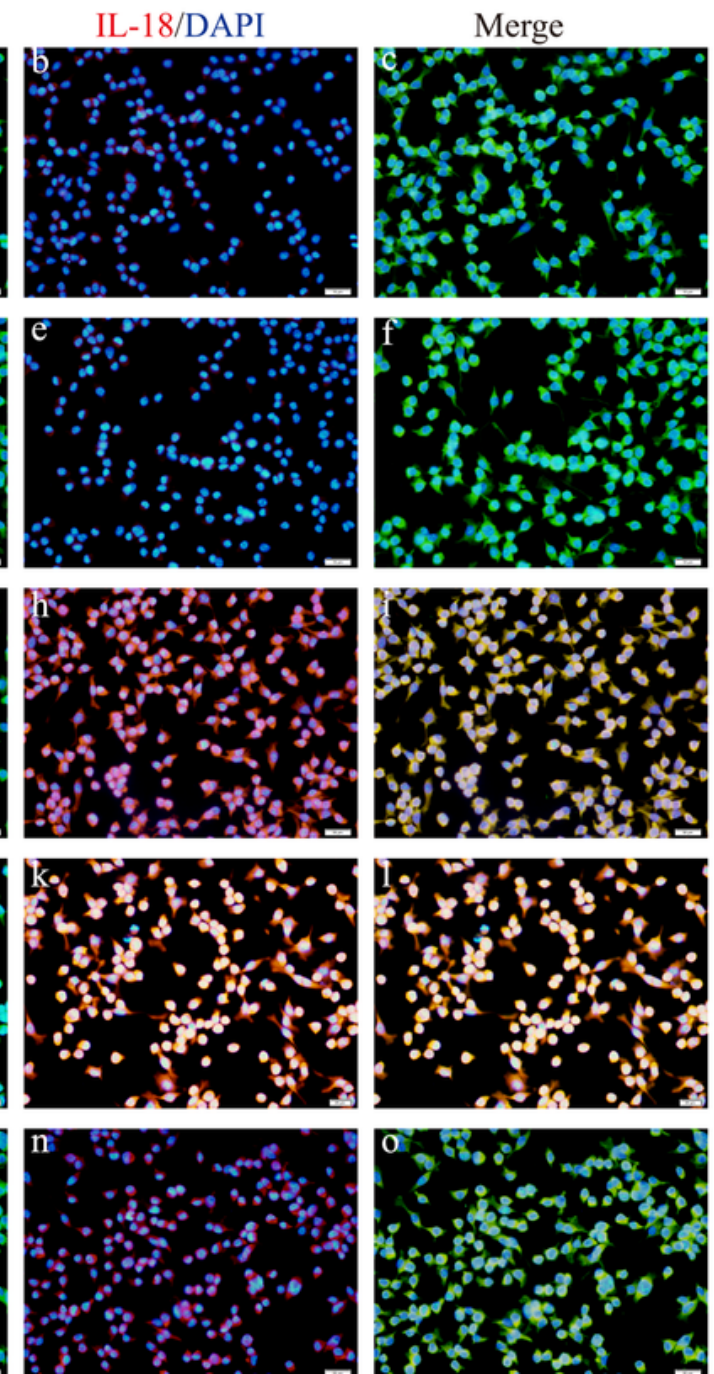

Figure 12

15\% CO2 increased IL-18 expression via inducing ROS overproduction in hypoxic BV-2 cells $(n=4)$. A Immunoreactive bands of IL-18 (17 kDa) and $\beta$-actin (42 kDa). B There is an interaction effect between $0.2 \% 02$ treatment and 15\% CO2 treatment $(P<0.01)$. C Simple effects analyses show increased protein expression levels of IL-18 in Hypoxia group ( $\left.{ }^{\mathrm{P}}<0.05\right)$, but not in $\mathrm{HC}$ group (ns $\mathrm{P}>0.05$ ) compared with Control group. Hypoxia + HC group shows the highest expression levels of IL-18 as compared with 
Hypoxia group ( ${ }^{\star \star} \mathrm{P}<0.01$ ) and $\mathrm{HC}$ group ( ${ }^{\star \star} \mathrm{P}<0.01$ ). Additionally, the protein expression of IL-18 is significantly suppressed with NAC pretreatment in BV-2 microglial cells (** $P<0.01)$. D

Immunofluorescence images showing Iba1+ microglia (a, d, g, j, m, green), IL-18 labeling (b, e, h, k, n, red), and their co-localization ( $\mathrm{x}, \mathrm{f}, \mathrm{i}, \mathrm{I}, \mathrm{o})$. Enhanced IL-18 immunofluorescence is evident in Hypoxia group, but not in $\mathrm{HC}$ group compared with Control group. Hypoxia $+\mathrm{HC}$ group displays the strongest IL-18 fluorescence when compared with Hypoxia group and HC group. IL-18 fluorescence is markedly reduced with NAC pretreatment (2 mM) in BV-2 microglial cells. Scale bars: $10 \mu \mathrm{m}$. IL-18, interleukin-18; ns, nonsignificant; $\mathrm{HC}$ group, high concentration of carbon dioxide group.

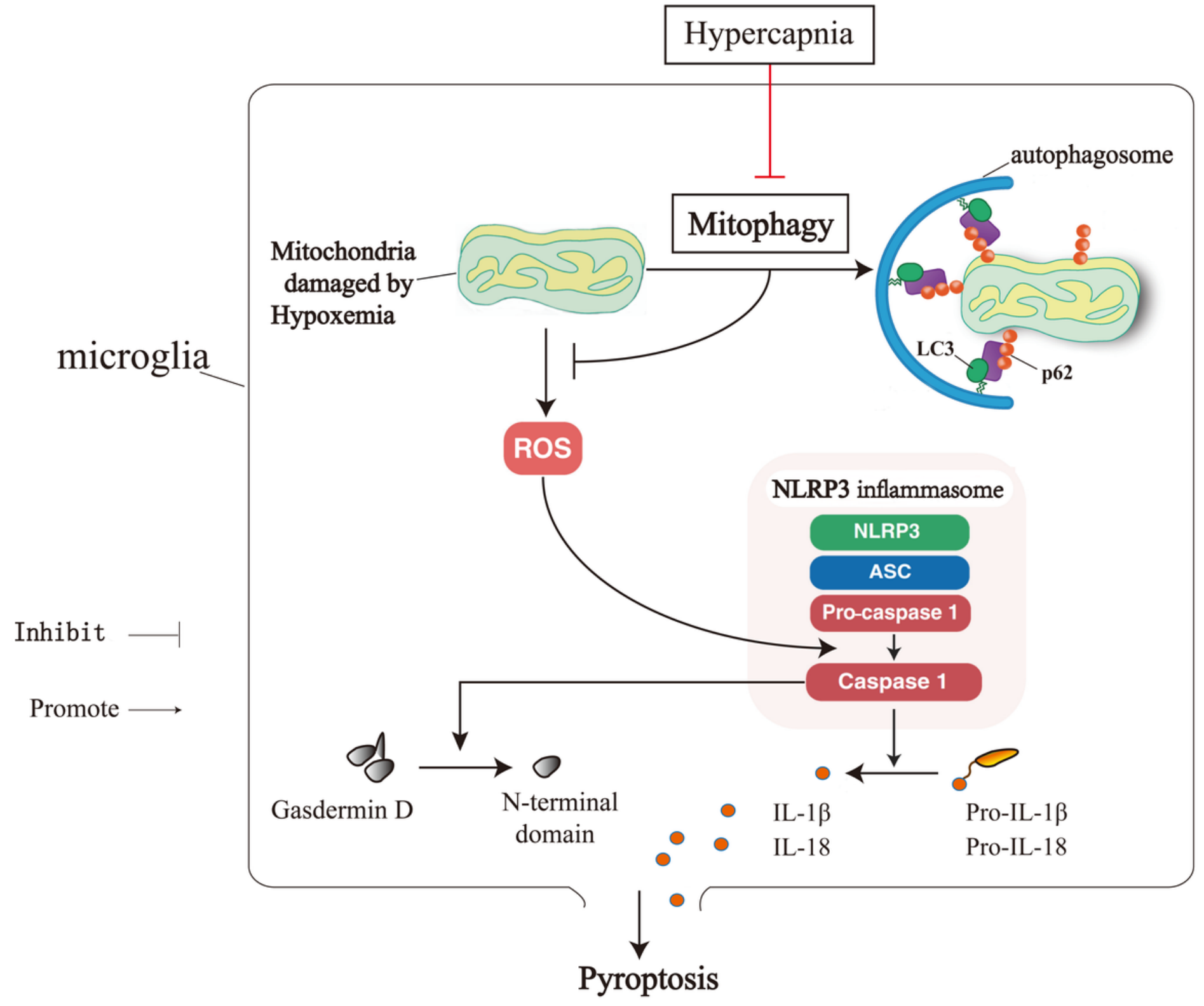

\section{Figure 13}

An illustration demonstrates that the molecular mechanism of hypercapnia promoting microglial pyroptosis via inhibiting mitophagy. 